\title{
Research Article \\ Effect of Rotation on Wave Propagation in Hollow Poroelastic Circular Cylinder
}

\author{
S. M. Abo-Dahab, ${ }^{1,2}$ A. M. Abd-Alla, ${ }^{1,3}$ and S. Alqosami ${ }^{1}$ \\ ${ }^{1}$ Mathematics Department, Faculty of Science, Taif University, Taif 888, Saudi Arabia \\ ${ }^{2}$ Mathematics Department, Faculty of Science, South Valley University, Qena 83523, Egypt \\ ${ }^{3}$ Mathematics Department, Faculty of Science, Sohag University, Egypt \\ Correspondence should be addressed to S. Alqosami; soo.s00@hotmail.com
}

Received 26 March 2014; Revised 10 May 2014; Accepted 11 May 2014; Published 25 September 2014

Academic Editor: Kim M. Liew

Copyright (C) 2014 S. M. Abo-Dahab et al. This is an open access article distributed under the Creative Commons Attribution License, which permits unrestricted use, distribution, and reproduction in any medium, provided the original work is properly cited.

\begin{abstract}
The objective of this paper is to study the effect of rotation on the wave propagation in an infinite poroelastic hollow circular cylinder. The frequency equation for poroelastic hollow circular cylinder is obtained when the boundaries are stress free and is examined numerically. The frequency, phase velocity, and attenuation coefficient are calculated for a pervious surface for various values of rotation, wave number, and thickness of the cylinder which are presented for nonaxial symmetric vibrations for a pervious surface. The dispersion curves are plotted for the poroelastic elastic behavior of the poroelastic material. Results are discussed for poroelastic material. The results indicate that the effect of rotation, wave number, and thickness on the wave propagation in the hollow poroelastic circular cylinder is very pronounced.
\end{abstract}

\section{Introduction}

The study of wave propagation over a continuous medium is of practical importance in the fields of engineering, medicine, and bioengineering. Application of the poroelastic materials in medical fields such as orthopedic, dental, and cardiovascular is well known. In orthopedics, wave propagation over bone is used in monitoring the rate of fracture healing. There are two types of osseous tissue such as cancellous or trabecular and compact or cortical bone, which are of different materials, with respect to their mechanical behavior. In macroscopic terms, the percentage of porosity in the cortical bone is 3$5 \%$, whereas, in the trabecular or cancellous, the percentage of porosity is up to $90 \%$ [1]. In fact, in a recent article, Ahmed and Abd-Alla [1] investigated the electromechanical wave propagation in a cylindrical poroelastic bone with cavity. Analytical solution of electromechanical wave propagation in long bones has been obtained by El-Naggar et al. [2]. AbdAlla et al. [3] studied the wave propagation modeling in cylindrical human long wet bones with cavity. Hart [4] investigated the theoretical study of the influence of bone maturation rate on surface remodeling predictions. Qin et al. [5] studied the thermoelectroelastic solutions for surface bone remodeling under axial and transverse loads. Martínez et al. [6] discussed the external bone remodeling through boundary elements and damage mechanics. Computational simulation of simultaneous cortical and trabecular bone change in human proximal femur during bone remodeling has been investigated by Jang and Kim [7]. Tsili [8] studied the theoretical solutions for internal bone remodeling of diaphyseal shafts using adaptive elasticity theory. Cowin and Firoozbakhsh [9] investigated the bone remodeling of diaphyseal surfaces under constant load: theoretical predictions, a contribution to the mechanics and thermodynamics of surface growth. Application to bone external remodeling has been studied by Ganghoffer [10]. Sims and Gooi [11] investigated bone remodeling: multiple cellular interactions required for coupling of bone formation and resorption. Zumsande et al. [12] discussed the general analysis of mathematical models for bone remodeling. Malachanne et al. [13] studied the numerical model of bone remodeling sensitive to loading frequency through a poroelastic behavior and internal fluid movements. A model for mechanical adaptation of trabecular bone incorporating cellular accommodation and effects of microdamage 
and disuse has been studied by Vahdati and Rouhi [14]. Hazelwood et al. [15] investigated the mechanistic model for internal bone remodeling exhibiting different dynamic responses in disuse and overload. Qu et al. [16] studied the hypothetical mechanism of bone remodeling and modeling under electromagnetic loads. Papathanasopoulou et al. [17] investigated the poroelastic bone model for internal remodeling. Isotropic continuum damage/repair model for alveolar bone remodeling has been studied by Mengoni and Ponthot [18]. Boyle and Kim [19] investigated the three-dimensional microlevel computational study of Wolff's law via trabecular bone remodeling in the human proximal femur using design space topology optimization. Wang et al. [20] studied the theoretical analysis of alendronate and risedronate effects on canine vertebral remodeling and microdamage. A physiologically based mathematical model of integrated calcium homeostasis and bone remodeling has been discussed by Peterson and Riggs [21]. Qin and Ye [22] studied the thermoelectroelas-tic solutions for internal bone remodeling investigated under axial and transverse loads. Boyle and Kim [23] studied the comparison of different hip prosthesis shapes considering microlevel bone remodeling and stress-shielding criteria using three-dimensional design space topology optimization. Cowin and Van Buskirk [24] investigated surface bone remodeling induced by a medullary pin. Biot [25] explained the general theory of three-dimensional consolidation. Hegedus and Cowin [26] studied bone remodeling II, small strain adaptive elasticity. The extensive literature on the topic is now available and we can only mention a few recent interesting investigations in [27-33]. Recently, AbdAlla and Abo-Dahab [34] investigated magnetic field effect on poroelastic bone model for internal remodeling.

In the present analysis, the free vibrations of an infinite hollow poroelastic circular cylinder are studied employing general displacement components in cylindrical polar coordinates, following Biot's [35] theory. The general frequency hollow cylinder is homogeneous and isotropic. Degenerate cases of the general frequency equation of pervious surfaces, when the longitudinal wave number $k$ is considered. The numerical result displayed by figures and the physical meaning are explained. The results and discussions presented in this study may be helpful to further understand wave propagation in hollow poroelastic circular cylinder.

\section{Governing Equations}

Let us consider that the equations of motion of a homogeneous, isotropic poroelastic solid in the presence of dissipation $b$ are

$$
\begin{aligned}
& (A+N) \\
& \times\left[\frac{\partial^{2} u_{r}}{\partial r^{2}}+\frac{1}{r} \frac{\partial u r}{\partial r}+\frac{1}{r} \frac{\partial^{2} u_{\theta}}{\partial r \partial \theta}\right. \\
& \left.\quad-\frac{1}{r^{2}} \frac{\partial u_{\theta}}{\partial \theta}+\frac{\partial^{2} u_{z}}{\partial r \partial z}-\frac{1}{r} u_{r}\right]
\end{aligned}
$$

$$
\begin{aligned}
&+N[ \frac{\partial^{2} u_{r}}{\partial r^{2}}+\frac{1}{r^{2}} \frac{\partial^{2} u_{r}}{\partial \theta^{2}}+\frac{\partial^{2} u_{r}}{\partial z^{2}} \\
&\left.+\frac{1}{r} \frac{\partial u r}{\partial r}-\frac{2}{r^{2}} \frac{\partial u_{\theta}}{\partial \theta}-\frac{2}{r} u_{r}\right] \\
&+Q[ \frac{\partial^{2} v_{r}}{\partial r^{2}}-\frac{v_{r}}{r^{2}}+\frac{1}{r} \frac{\partial^{2} v_{\theta}}{\partial r \partial \theta} \\
&\left.+\frac{\partial^{2} v_{z}}{\partial r \partial z}-\frac{1}{r^{2}} \frac{\partial v_{\theta}}{\partial \theta}+\frac{1}{r} \frac{\partial v r}{\partial r}\right] \\
&=\frac{\partial^{2}}{\partial t^{2}}\left(\rho_{11} u_{r}+\rho_{12} v_{r}\right)-\rho_{11} \Omega^{2} u_{r}+b \frac{\partial}{\partial t}\left(u_{r}-v_{r}\right)
\end{aligned}
$$$$
(A+N)
$$$$
\times\left[\frac{1}{r} \frac{\partial^{2} u_{r}}{\partial r \partial \theta}+\frac{1}{r} \frac{\partial^{2} u_{z}}{\partial \theta \partial z}+\frac{1}{r^{2}} \frac{\partial u r}{\partial \theta}+\frac{1}{r^{2}} \frac{\partial^{2} u_{\theta}}{\partial r^{2}}\right]
$$$$
+N\left[\frac{2}{r^{2}} \frac{\partial u r}{\partial \theta}+\frac{1}{r^{2}} \frac{\partial^{2} u_{\theta}}{\partial \theta^{2}}\right.
$$$$
\left.+\frac{\partial^{2} u_{\theta}}{\partial r^{2}}+\frac{1}{r} \frac{\partial u_{\theta}}{\partial r}-\frac{1}{r} u_{\theta}+\frac{\partial^{2} u_{\theta}}{\partial z^{2}}\right]
$$$$
+\frac{Q}{r}\left[\frac{\partial^{2} v_{\theta}}{\partial r \partial \theta}+\frac{1}{r^{2}} \frac{\partial^{2} v_{\theta}}{\partial \theta^{2}}+\frac{1}{r} \frac{\partial v_{r}}{\partial \theta}+\frac{\partial^{2} v_{z}}{\partial \theta \partial z}\right]
$$$$
=\frac{\partial^{2}}{\partial t^{2}}\left(\rho_{11} u_{\theta}+\rho_{12} v_{\theta}\right)+b \frac{\partial}{\partial t}\left(u_{\theta}-v_{\theta}\right)
$$$$
(A+N)\left[\frac{\partial^{2} u_{r}}{\partial r \partial z}+\frac{1}{r} \frac{\partial^{2} u_{\theta}}{\partial \theta \partial z}+\frac{1}{r} \frac{\partial u r}{\partial z}+\frac{\partial^{2} u_{z}}{\partial z^{2}}\right]
$$$$
+N\left[\frac{\partial^{2} u_{z}}{\partial z^{2}}+\frac{\partial^{2} u_{z}}{\partial r^{2}}+\frac{1}{r^{2}} \frac{\partial^{2} u_{z}}{\partial \theta^{2}}+\frac{1}{r} \frac{\partial u_{z}}{\partial z}\right]
$$$$
+Q\left[\frac{\partial^{2} v_{r}}{\partial r \partial z}+\frac{1}{r^{2}} \frac{\partial^{2} v_{\theta}}{\partial \theta \partial z}+\frac{1}{r} \frac{\partial v_{r}}{\partial z}+\frac{\partial^{2} v_{z}}{\partial^{2} z}\right]
$$$$
=\frac{\partial^{2}}{\partial t^{2}}\left(\rho_{11} u_{z}+\rho_{12} v_{z}\right)-\rho_{11} \Omega^{2} u_{z}+b \frac{\partial}{\partial t}\left(u_{z}-v_{z}\right)
$$$$
Q\left(\frac{\partial^{2} u_{r}}{\partial r^{2}}+\frac{1}{r} \frac{\partial u r}{\partial r}+\frac{1}{r} \frac{\partial^{2} u_{\theta}}{\partial r \partial \theta}\right.
$$$$
\left.-\frac{1}{r^{2}} \frac{\partial u_{\theta}}{\partial \theta}+\frac{\partial^{2} u_{z}}{\partial r \partial z}-\frac{1}{r} u_{r}\right)
$$$$
+R\left[\frac{\partial^{2} v_{r}}{\partial r^{2}}-\frac{v_{r}}{r^{2}}+\frac{1}{r} \frac{\partial^{2} v_{\theta}}{\partial r \partial \theta}\right.
$$$$
\left.+\frac{\partial^{2} v_{z}}{\partial r \partial z}-\frac{1}{r^{2}} \frac{\partial v_{\theta}}{\partial \theta}+\frac{1}{r} \frac{\partial v r}{\partial r}\right]
$$ 


$$
\begin{aligned}
= & \frac{\partial^{2}}{\partial t^{2}}\left(\rho_{12} u_{r}+\rho_{22} v_{r}\right)-b \frac{\partial}{\partial t}\left(u_{r}-v_{r}\right), \\
Q & {\left[\frac{1}{r} \frac{\partial^{2} u_{r}}{\partial r \partial \theta}+\frac{1}{r} \frac{\partial^{2} u_{z}}{\partial \theta \partial z}+\frac{1}{r^{2}} \frac{\partial u r}{\partial \theta}+\frac{1}{r^{2}} \frac{\partial^{2} u_{\theta}}{\partial r^{2}}\right] } \\
& +\frac{R}{r}\left[\frac{\partial^{2} v_{\theta}}{\partial r \partial \theta}+\frac{1}{r} \frac{\partial^{2} v_{\theta}}{\partial \theta^{2}}+\frac{1}{r} \frac{\partial v_{r}}{\partial \theta}+\frac{\partial^{2} v_{z}}{\partial \theta \partial z}\right] \\
= & \frac{\partial^{2}}{\partial t^{2}}\left(\rho_{12} u_{\theta}+\rho_{22} v_{\theta}\right)-b \frac{\partial}{\partial t}\left(u_{\theta}-v_{\theta}\right), \\
Q & {\left[\frac{\partial^{2} u_{r}}{\partial r \partial z}+\frac{1}{r} \frac{\partial^{2} u_{\theta}}{\partial \theta \partial z}+\frac{1}{r} \frac{\partial u r}{\partial z}+\frac{\partial^{2} u_{z}}{\partial z^{2}}\right] } \\
& +R\left[\frac{\partial^{2} v_{r}}{\partial r \partial z}+\frac{1}{r^{2}} \frac{\partial^{2} v_{\theta}}{\partial \theta \partial z}+\frac{1}{r} \frac{\partial v_{r}}{\partial z}+\frac{\partial^{2} v_{z}}{\partial^{2} z}\right] \\
= & \frac{\partial^{2}}{\partial t^{2}}\left(\rho_{12} u_{z}+\rho_{22} v_{z}\right)-b \frac{\partial}{\partial t}\left(u_{z}-v_{z}\right),
\end{aligned}
$$

where $\nabla^{2}$ is the Laplace operator, $\vec{u}=\left(u_{r}, u_{\theta}, u_{z}\right), \vec{v}=$ $\left(v_{r}, v_{\theta}, v_{z}\right)$ are displacements of solid and liquid, respectively, $e$ and $\varepsilon$ are the dilatations of solid and liquid, $A, N, Q$, and $R$ are all poroelastic constants, and $\rho_{i j}(i, j=1,2)$ are the mass coefficients following Biot [35].

The stress-strain equations for an isotropic poroelastic material of solid and liquid are given as

$$
\begin{aligned}
\sigma_{r r} & =2 N E_{r r}+A E+Q \varepsilon, \\
\sigma_{\theta \theta} & =2 N E_{\theta \theta}+A E+Q \varepsilon, \\
\sigma_{z z} & =2 N E_{z z}+A E+Q \varepsilon, \\
\tau_{\theta z} & =N E_{\theta z}, \\
\tau_{z r} & =N E_{z r}, \\
\tau_{r \theta} & =N E r_{\theta}, \\
s & =Q E+R \varepsilon .
\end{aligned}
$$

\section{Solution of the Problem}

Let us consider $(r, \theta, z)$ to be the cylindrical polar coordinates. Consider a homogeneous, isotropic, poroelastic cylinder with inner and outer radii being $r_{1}$ and $r_{2}$, respectively, so that the thickness of poroelastic cylinder is $h\left[=\left(r_{2}-r_{1}\right)>0\right]$, whose axis is in the direction of $z$-axis; let

$$
\begin{aligned}
& \vec{v}=\underline{\nabla} \phi_{2}+\underline{\nabla} \wedge \vec{\psi}_{2}, \\
& \vec{u}=\underline{\nabla} \phi_{1}+\underline{\nabla} \wedge \vec{\psi}_{1},
\end{aligned}
$$

where $\vec{u}, \vec{v}$ are displacements of solid and liquid, respectively, $\phi_{1}, \phi_{2}$ are scalar potentials, and $\vec{\psi}_{1}, \vec{\psi}_{2}$ are vector potentials.
Assuming that

$$
\begin{gathered}
\vec{\Psi}_{1}=\left(h_{r}, h_{\theta}, h_{z}\right), \\
\vec{\Psi}_{2}=\left(H_{r}, H_{\theta}, H_{z}\right),
\end{gathered}
$$

from (1) and (3), we get

$$
\begin{gathered}
P \nabla^{2} \emptyset_{1}+Q \nabla^{2} \emptyset_{2}=\left(\rho_{11} \ddot{\emptyset}_{1}\left(1+\Omega^{2}\right)+\rho_{12} \ddot{\emptyset}_{2}\right)+b\left(\dot{\emptyset}_{1}-\dot{\emptyset}_{2}\right) \\
Q \nabla^{2} \emptyset_{1}+R \nabla^{2} \emptyset_{2}=\left(\rho_{12} \ddot{\emptyset}_{1}+\rho_{22} \ddot{\emptyset}_{2}\right)-b\left(\dot{\emptyset}_{1}-\dot{\emptyset}_{2}\right) \\
0=\left(\rho_{12} \overrightarrow{\ddot{\Psi}}_{1}+\rho_{22} \vec{\Psi}_{2}\right)-b\left(\overrightarrow{\dot{\Psi}}_{1}-\overrightarrow{\dot{\Psi}}_{2}\right) \\
N \nabla^{2} \vec{\Psi}_{1} \\
=\left(\rho_{11} \vec{\Psi}_{1}\left(1+\Omega^{2}\right)+\rho_{12} \vec{\Psi}_{2}\right)+b\left(\overrightarrow{\dot{\Psi}}_{1}-\overrightarrow{\dot{\Psi}}_{2}\right)
\end{gathered}
$$

where $P=A+2 N$.

Assuming that

$$
\begin{aligned}
& \emptyset_{1}=f_{1}(r) \cos (n \theta) e^{i(k z+w t)}, \\
& \emptyset_{2}=f_{2}(r) \cos (n \theta) e^{i(k z+w t)}, \\
& h_{r}=g_{r}(r) \sin (n \theta) e^{i(k z+w t)}, \\
& h_{\theta}=g_{\theta}(r) \cos (n \theta) e^{i(k z+w t)}, \\
& h_{z}=g_{z}(r) \sin (n \theta) e^{i(k z+w t)}, \\
& H_{r}=G_{r}(r) \sin (n \theta) e^{i(k z+w t)}, \\
& H_{\theta}=G_{\theta}(r) \cos (n \theta) e^{i(k z+w t)}, \\
& H_{z}=G_{z}(r) \sin (n \theta) e^{i(k z+w t)},
\end{aligned}
$$

where $k$ is the axial wave number, $n$ is an integer number of waves around the circumference or also known as angular wave number, and $w$ is circular frequency. From the second and third equations of (5) with (4), when the first two equations of (5) remain the same, they are reduced to

$$
\begin{aligned}
& P \nabla^{2} \emptyset_{1}+Q \nabla^{2} \emptyset_{2} \\
& \quad=\left(\rho_{11} \ddot{\emptyset}_{1}\left(1+\Omega^{2}\right)+\rho_{12} \ddot{\emptyset}_{2}\right)+b\left(\dot{\emptyset}_{1}-\dot{\emptyset}_{2}\right), \\
& Q \nabla^{2} \emptyset_{1}+R \nabla^{2} \emptyset_{2} \\
& \quad=\left(\rho_{12} \ddot{\emptyset}_{1}+\rho_{22} \ddot{\emptyset}_{2}\right)-b\left(\dot{\emptyset}_{1}-\dot{\emptyset}_{2}\right),
\end{aligned}
$$




$$
\begin{aligned}
N & \left(\nabla^{2} h_{r}-\frac{h_{r}}{r^{2}}-\frac{2}{r^{2}} \frac{\partial h_{\theta}}{\partial \theta}\right) \\
& =\left(\left(\rho_{11}+\Omega^{2}\right) \ddot{h}_{r}+\rho_{12} \ddot{H}_{r}\right)+b\left(\dot{h}_{r}-\dot{H}_{r}\right), \\
N & \left(\nabla^{2} h_{\theta}-\frac{h_{\theta}}{r^{2}}+\frac{2}{r^{2}} \frac{\partial h_{r}}{\partial \theta}\right) \\
& =\left(\left(\rho_{11}+\Omega^{2}\right) \ddot{h}_{\theta}+\rho_{12} \ddot{H}_{\theta}\right)+b\left(\dot{h}_{\theta}-\dot{H}_{\theta}\right), \\
N & \left(\nabla^{2} h_{z}\right) \\
& =\left(\left(\rho_{11}+\Omega^{2}\right) \ddot{h}_{z}+\rho_{12} \ddot{H}_{z}\right)+b\left(\dot{h}_{z}-\dot{H}_{z}\right), \\
0 & =\left(\rho_{12} \ddot{h}_{r}+\rho_{22} \ddot{H}_{r}\right)-b\left(\dot{h}_{r}-\dot{H}_{r}\right), \\
0 & =\left(\rho_{12} \ddot{h}_{\theta}+\rho_{22} \ddot{H}_{\theta}\right)-b\left(\dot{h}_{\theta}-\dot{H}_{\theta}\right), \\
0 & =\left(\rho_{12} \ddot{h}_{z}+\rho_{22} \ddot{H}_{z}\right)-b\left(\dot{h}_{z}-\dot{H}_{z}\right) .
\end{aligned}
$$

From (7) and (6), we obtain

$$
\begin{aligned}
& p \Delta f_{1}+Q \Delta f_{2}=-w^{2}\left[k_{11} f_{1}+k_{12} f_{2}\right], \\
& Q \Delta f_{1}+R \Delta f_{2}=-w^{2}\left[k_{12} f_{1}+k_{22} f_{2}\right], \\
& N\left[\Delta g_{r}-\frac{g_{r}}{r^{2}}-\frac{2 n}{r^{2}} g_{\theta}\right]=-w^{2}\left[k_{11}^{\prime} g_{r}+k_{12} G_{r}\right], \\
& N\left[\Delta g_{\theta}-\frac{g_{\theta}}{r^{2}}-\frac{2 n}{r^{2}} g_{r}\right]=-w^{2}\left[k_{11}^{\prime} g_{\theta}+k_{12} G_{\theta}\right], \\
& N \Delta g_{3}=-w^{2}\left[k_{11}^{\prime} g_{z}+k_{12} G_{z}\right], \\
& 0=-w^{2}\left[k_{12} g_{r}+k_{22} G_{r}\right], \\
& 0=-w^{2}\left[k_{12} g_{\theta}+k_{22} G_{\theta}\right], \\
& 0=-w^{2}\left[k_{12} g_{z}+k_{22} G_{z}\right],
\end{aligned}
$$

where

$$
\begin{aligned}
& \Delta=\frac{d^{2}}{d r^{2}}+\frac{1}{r} \frac{d}{d r}-\frac{n^{2}}{r^{2}}-k^{2}, \\
& k_{11}=\rho_{11}\left(1-\Omega^{2}\right)-\frac{i b}{w}, \\
& k_{12}=\rho_{12}+\frac{i b}{w}, \quad k_{22}=\rho_{22}-\frac{i b}{w}, \\
& k_{11}^{\prime}=\rho_{11}\left(1+\Omega^{2}\right)-\frac{i b}{w} .
\end{aligned}
$$

The general solution of (8) can be obtained in terms of the Bessel function of the first and second kind $J$ and $Y$ depending on its arguments $\propto_{1} r, \propto_{2} r, \propto_{3} r$.
From (8), we obtain

$$
\begin{gathered}
f_{1}=c_{1} J_{n}\left(\propto_{1} r\right)+c_{2} Y_{n}\left(\propto_{1} r\right)+c_{3} J_{n}\left(\propto_{2} r\right)+c_{4} Y_{n}\left(\propto_{2} r\right), \\
g_{3}(r)=A_{3} J_{n}\left(\alpha_{3} r\right)+B_{3} Y_{n}\left(\alpha_{3} r\right), \\
2 g_{1}=g_{r}-g_{\theta}=2 A_{1} J_{n+1}\left(\propto_{3} r\right)+2 B_{1} Y_{n+1}\left(\propto_{3} r\right), \\
2 g_{2}=g_{r}+g_{\theta}=2 A_{2} J_{n-1}\left(r \alpha_{3}\right)+2 B_{2} Y_{n-1}\left(r \alpha_{3}\right),
\end{gathered}
$$

where $J_{n}$ is Bessel function of the first kind and order $n$ and $Y_{n}$ is the Bessel function of the second kind and order $n$. Consider that

$$
\begin{aligned}
& \propto_{1}^{2}=\zeta_{1}^{2}-k^{2}+\Omega^{2}, \\
& \propto_{2}^{2}=\zeta_{2}^{2}-k^{2}+\Omega^{2}, \\
& \propto_{3}^{2}=\zeta_{3}^{2}-k^{2}-\Omega^{2}
\end{aligned}
$$

are positive or negative and

$$
\omega^{2}=\zeta_{i}^{2} v_{i}^{2} \quad(i=1,2,3)
$$

where $v_{r}, v_{\theta}$ are the dilatational wave velocities of first and second kind, respectively, and $v_{z}$ is shear wave velocity.

The gauge invariance property, following the analysis of [36], is used to eliminate two integration constants from (10). Any one of the potential functions $g_{1}, g_{2}$, or $g_{3}$ can be set equal to zero, without loss of generality of the solution. Setting $g_{2}=0$, we can obtain

$$
g_{r}=-g_{\theta}=g_{1} \text {. }
$$

The displacement vector of solid $\vec{u}=\left(u_{r}, u_{\theta}, u_{z}\right)$ with the help of (3) and (4) is given by

$$
\begin{aligned}
& u_{r}=\frac{\partial \emptyset_{1}}{\partial r}+\frac{1}{r} \frac{\partial h_{z}}{\partial \theta}-\frac{\partial h_{\theta}}{\partial z}, \\
& u_{\theta}=\frac{1}{r} \frac{\partial \emptyset_{1}}{\partial \theta}+\frac{\partial h_{r}}{\partial z}-\frac{\partial h_{z}}{\partial r}, \\
& u_{z}=\frac{\partial \emptyset_{1}}{\partial z}+\frac{\partial h_{\theta}}{\partial r}+\frac{h_{\theta}}{r}-\frac{1}{r} \frac{\partial h_{r}}{\partial \theta} .
\end{aligned}
$$

Substituting from (6) into (14), the displacement components of solid are

$$
\begin{gathered}
u_{r}=\left[f_{1}^{\prime}+\frac{n}{r} g_{z}-i k g_{\theta}\right] \cos (n \theta) e^{i(k z+\omega t)}, \\
u_{\theta}=\left[-\frac{n}{r} f_{1}+i k g_{r}-g_{z}^{\prime}\right] \sin (n \theta) e^{i(k z+\omega t)}, \\
u_{z}=\left[i k f_{1}+g_{\theta}^{\prime}+g_{\theta}-\frac{n}{r} g_{r}\right] \cos (n \theta) e^{i(k z+\omega t)} .
\end{gathered}
$$

Substituting from (13) into (15), the solid displacement is

$$
u_{r}=\left[f_{1}^{\prime}+\frac{n}{r} g_{3}-i k g_{1}\right] \cos (n \theta) e^{i(k z+\omega t)}
$$




$$
\begin{gathered}
u_{\theta}=\left[-\frac{n}{r} f_{1}+i k g_{1}-g_{3}^{\prime}\right] \sin (n \theta) e^{i(k z+\omega t)}, \\
u_{z}=\left[i k f_{1}+g_{1}^{\prime}-\frac{(n+1)}{r} g_{1}\right] \cos (n \theta) e^{i(k z+\omega t)},
\end{gathered}
$$

where "prime" over a quantity denotes differentiation with respect to $r, \beta_{1}, \beta_{2}, \beta_{3}$ being the absolute values of $\alpha_{1}, \alpha_{2}, \alpha_{3}$, respectively.

The dilatations of solid and liquid media are

$$
\begin{aligned}
& e=\Delta f_{1}(r) \cos (n \theta) e^{i(k z+\omega t)}, \\
& \epsilon=\Delta f_{2}(r) \cos (n \theta) e^{i(k z+\omega t)} .
\end{aligned}
$$

Substituting from (16) into strain displacement relations and then using (2), the stresses $\sigma_{i j}$ and the liquid pressures are

$$
\begin{aligned}
& \sigma_{r r}+s=\left[c_{1} M_{11}(r)+c_{2} M_{12}(r)\right. \\
& +c_{3} M_{13}(r)+c_{4} M_{14}(r)+A_{3} M_{15}(r) \\
& \left.+B_{3} M_{16}(r)+A_{1} M_{17}(r)+B_{1} M_{18}(r)\right] \\
& \times \cos (n \theta) e^{i(k z+\omega t)}, \\
& \sigma_{r \theta}=\left[c_{1} M_{21}(r)+c_{2} M_{22}(r)\right. \\
& +c_{3} M_{23}(r)+c_{4} M_{24}(r)+A_{3} M_{25}(r) \\
& \left.+B_{3} M_{26}(r)+A_{1} M_{27}(r)+B_{1} M_{28}(r)\right] \\
& \times \sin (n \theta) e^{i(k z+\omega t)}, \\
& \sigma_{r z}=\left[c_{1} M_{31}(r)+c_{2} M_{32}(r)\right. \\
& +c_{3} M_{33}(r)+c_{4} M_{34}(r)+A_{3} M_{35}(r) \\
& \left.+B_{3} M_{36}(r)+A_{1} M_{37}(r)+B_{1} M_{38}(r)\right] \\
& \times \cos (n \theta) e^{i(k z+\omega t)}, \\
& s=\left[c_{1} M_{41}(r)+c_{2} M_{42}(r)\right. \\
& \left.+c_{3} M_{43}(r)+c_{4} M_{44}(r)\right] \\
& \times \cos (n \theta) e^{i(k z+\omega t)}, \\
& \frac{\partial s}{\partial r}=\left[c_{1} M_{41}(r)+c_{2} M_{42}(r)\right. \\
& \left.+c_{3} M_{43}(r)+c_{4} M_{44}(r)\right] \\
& \times \cos (n \theta) e^{i(k z+\omega t)},
\end{aligned}
$$

where the coefficients $M_{i j}(r)$ are given as

$$
\begin{aligned}
& M_{11}(r)=\left\{2 N\left[\frac{n(n-1)}{r^{2}}-\alpha_{1}^{2}\right]\right. \\
& \left.+\left[(Q+R) \delta_{1}^{2}-(A+Q)\right]\left(k^{2}+\alpha_{1}^{2}\right)\right\} \\
& \times J_{n}\left(\beta_{1} r\right)+\frac{2 N \beta_{1}}{r} J_{n+1}\left(\beta_{1} r\right), \\
& M_{12}(r)=\left\{2 N\left[\frac{n(n-1)}{r^{2}}-\alpha_{1}^{2}\right]\right. \\
& \left.+\left[(Q+R) \delta_{1}^{2}-(A+Q)\right]\left(k^{2}+\alpha_{1}^{2}\right)\right\} \\
& \times Y_{n}\left(\beta_{1} r\right)+\frac{2 N \beta_{1}}{r} Y_{n+1}\left(\beta_{1} r\right), \\
& M_{13}(r)=\left\{2 N\left[\frac{n(n-1)}{r^{2}}-\alpha_{1}^{2}\right]\right. \\
& \left.+\left[(Q+R) \delta_{1}^{2}-(A+Q)\right]\left(k^{2}+\alpha_{1}^{2}\right)\right\} \\
& \times J_{n}\left(\beta_{1} r\right)+\frac{2 N \beta_{1}}{r} J_{n+1}\left(\beta_{1} r\right), \\
& M_{14}(r)=\left\{2 N\left[\frac{n(n-1)}{r^{2}}-\alpha_{1}^{2}\right]\right. \\
& \left.+\left[(Q+R) \delta_{1}^{2}-(A+Q)\right]\left(k^{2}+\alpha_{1}^{2}\right)\right\} \\
& \times Y_{n}\left(\beta_{1} r\right)+\frac{2 N \beta_{1}}{r} J_{n+1}\left(\beta_{1} r\right), \\
& M_{15}(r)=\frac{2 N n(n-1)}{r^{2}} J_{n}\left(\beta_{3} r\right)-\frac{2 N n \beta_{3}}{r} J_{n+1}\left(\beta_{3} r\right) \text {, } \\
& M_{16}(r)=\frac{2 N n(n-1)}{r^{2}} Y_{n}\left(\beta_{3} r\right)-\frac{2 N n \beta_{3}}{r} Y_{n+1}\left(\beta_{3} r\right) \text {, } \\
& M_{17}(r)=2 N i k \beta_{3} J_{n}\left(\beta_{3} r\right)-\frac{2 N(n+1) i k}{r} J_{n+1}\left(\beta_{3} r\right) \text {, } \\
& M_{18}(r)=2 N i k \beta_{3} Y_{n}\left(\beta_{3} r\right)-\frac{2 N(n+1) i k}{r} Y_{n+1}\left(\beta_{3} r\right) \text {, } \\
& M_{21}(r)=\frac{2 N n(1-n)}{r^{2}} Y_{n}\left(\beta_{1} r\right)+\frac{2 N n \beta_{1}}{r} J_{n+1}\left(\beta_{1} r\right), \\
& M_{22}(r)=\frac{2 N n(1-n)}{r^{2}} Y_{n}\left(\beta_{1} r\right)+\frac{2 N n \beta_{1}}{r} Y_{n+1}\left(\beta_{1} r\right) \text {, } \\
& M_{23}(r)=\frac{2 N n(1-n)}{r^{2}} J_{n}\left(\beta_{2} r\right)+\frac{2 N n \beta_{2}}{r} J_{n+1}\left(\beta_{2} r\right) \text {, } \\
& M_{24}(r)=\frac{2 N n(1-n)}{r^{2}} Y_{n}\left(\beta_{2} r\right)+\frac{2 N n \beta_{2}}{r} Y_{n+1}\left(\beta_{2} r\right) \text {, } \\
& M_{25}(r)=\left[\frac{2 N n(1-n)}{r^{2}}+N \alpha_{3}^{2}\right] J_{n}\left(\beta_{3} r\right) \\
& -\frac{2 N \beta_{3}}{r} J_{n+1}\left(\beta_{3} r\right)
\end{aligned}
$$




$$
\begin{aligned}
& M_{26}(r)=\left[\frac{2 N n(1-n)}{r^{2}}+N \alpha_{3}^{2}\right] Y_{n}\left(\beta_{3} r\right) \\
& -\frac{2 N \beta_{3}}{r} Y_{n+1}\left(\beta_{3} r\right) \text {, } \\
& M_{27}(r)=i k N \beta_{3} J_{n}\left(\beta_{3} r\right) \\
& -\frac{2 N(n+1) i k}{r} J_{n+1}\left(\beta_{3} r\right), \\
& M_{28}(r)=i k N \beta_{3} Y_{n}\left(\beta_{3} r\right) \\
& -\frac{2 N(n+1) i k}{r} Y_{n+1}\left(\beta_{3} r\right), \\
& M_{31}(r)=\frac{2 N n i k}{r} J_{n}\left(\beta_{1} r\right)-2 N i k \beta_{1} J_{n+1}\left(\beta_{1} r\right), \\
& M_{32}(r)=\frac{2 N n i k}{r} Y_{n}\left(\beta_{1} r\right)-2 N i k \beta_{1} Y_{n+1}\left(\beta_{1} r\right), \\
& M_{33}(r)=\frac{2 N n i k}{r} J_{n}\left(\beta_{2} r\right)-2 N i k \beta_{2} J_{n+1}\left(\beta_{2} r\right), \\
& M_{34}(r)=\frac{2 N n i k}{r} Y_{n}\left(\beta_{2} r\right)-2 N i k \beta_{2} Y_{n+1}\left(\beta_{2} r\right), \\
& M_{35}(r)=\frac{N n i k}{r} J_{n}\left(\beta_{3} r\right), \\
& M_{36}(r)=\frac{N n i k}{r} Y_{n}\left(\beta_{3} r\right), \\
& M_{37}(r)=\frac{-N n \beta_{3}}{r} J_{n}\left(\beta_{3} r\right)-N\left(k^{2}-\alpha_{3}^{2}\right) J_{n+1}\left(\beta_{3} r\right) \text {, } \\
& M_{38}(r)=\frac{-N n \beta_{3}}{r} Y_{n}\left(\beta_{3} r\right)-N\left(k^{2}-\alpha_{3}^{2}\right) Y_{n+1}\left(\beta_{3} r\right), \\
& M_{41}(r)=\left(R \delta_{1}^{2}-Q\right)\left(k^{2}+\alpha_{1}^{2}\right) J_{n}\left(\beta_{1} r\right),
\end{aligned}
$$

$$
\begin{gathered}
M_{42}(r)=\left(R \delta_{1}^{2}-Q\right)\left(k^{2}+\alpha_{1}^{2}\right) Y_{n}\left(\beta_{1} r\right), \\
M_{43}(r)=\left(R \delta_{2}^{2}-Q\right)\left(k^{2}+\alpha_{1}^{2}\right) J_{n}\left(\beta_{2} r\right), \\
M_{44}(r)=\left(R \delta_{2}^{2}-Q\right)\left(k^{2}+\alpha_{2}^{2}\right) Y_{n}\left(\beta_{2} r\right), \\
M_{45}(r)=0, \quad M_{46}(r)=0, \\
M_{47}(r)=0, \quad M_{48}(r)=0 .
\end{gathered}
$$

In (23),

$$
\begin{aligned}
\delta_{i}^{2}= & \frac{1}{\left(R k_{12}-Q k_{22}\right)} \\
& \times\left[\left(R k_{11}-Q k_{12}\right)-V_{i}^{-2}\left(P R-Q^{2}\right)\right] \\
& (i=1,2),
\end{aligned}
$$

where we considered

$$
\left|k v_{1}\right|<\omega
$$

\section{Boundary Conditions and Frequency Equation}

The boundary conditions for traction free inner and outer surfaces of the hollow poroelastic cylinder in case of a pervious surface are

$$
\begin{gathered}
\sigma_{r r}+s=0, \quad \sigma_{r \theta}=0, \quad \sigma_{r z}=0, \\
s=0, \quad \text { at } r=r_{1}, \quad r=r_{2} .
\end{gathered}
$$

Equations (18)-(21) together with (26) yield eight homogeneous equations for eight arbitrary constants $C_{1}, C_{2}, C_{3}, C_{4}$, $A_{3}, B_{3}, A_{1}$, and $B_{1}$. A nontrivial solution can be obtained when the determinant of coefficients vanishes. Thus, the frequency equation for a previous surface is

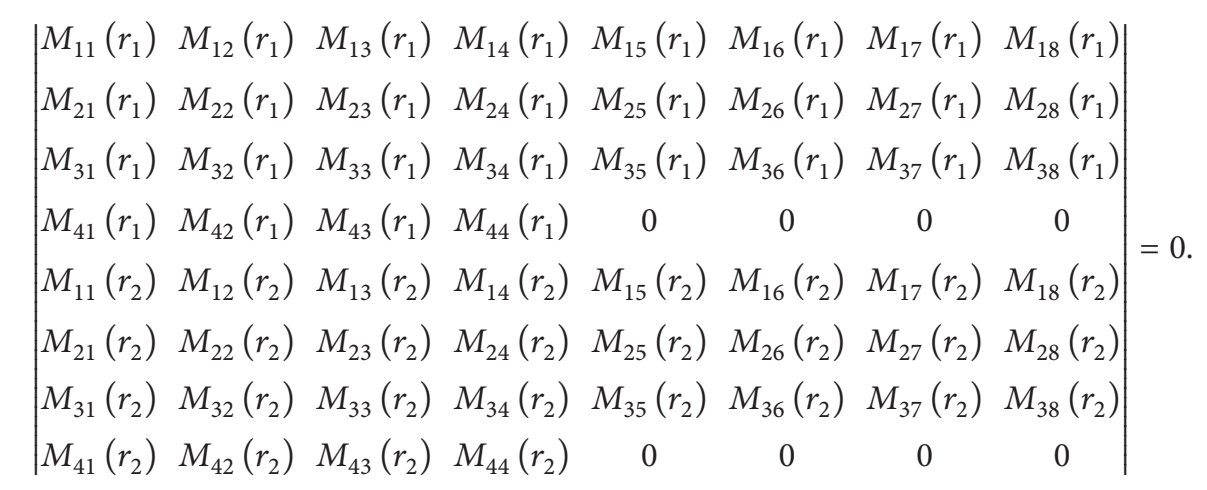

In (27), the element $M_{i j}(r)$ is defined in (23).

By ignoring the liquid effects in the frequency equation of a pervious surface (27), the results of purely elastic solid are obtained as a special case considered by [36]. Now, we consider the particular cases of the general frequency equations (27) when the axial and angular wave numbers vanish. 
4.1. Motion Independent of $z$. When the axial wave number $k$ is taken to be equal to zero, that is, by considering zeroth azimuthal mode, the frequency equation of a pervious surface
(28) degenerates into the product of two determinants as follows:

$$
D_{1} D_{2}=0
$$

where

$$
D_{1}=\left|\begin{array}{cccccc}
M_{11}^{\prime}\left(r_{1}\right) & M_{12}^{\prime}\left(r_{1}\right) & M_{13}^{\prime}\left(r_{1}\right) & M_{14}^{\prime}\left(r_{1}\right) & M_{15}^{\prime}\left(r_{1}\right) & M_{16}^{\prime}\left(r_{1}\right) \\
M_{21}^{\prime}\left(r_{1}\right) & M_{22}^{\prime}\left(r_{1}\right) & M_{23}^{\prime}\left(r_{1}\right) & M_{24}^{\prime}\left(r_{1}\right) & M_{25}^{\prime}\left(r_{1}\right) & M_{26}^{\prime}\left(r_{1}\right) \\
M_{41}^{\prime}\left(r_{1}\right) & M_{42}^{\prime}\left(r_{1}\right) & M_{43}^{\prime}\left(r_{1}\right) & M_{44}^{\prime}\left(r_{1}\right) & 0 & 0 \\
M_{11}^{\prime}\left(r_{2}\right) & M_{12}^{\prime}\left(r_{2}\right) & M_{13}^{\prime}\left(r_{2}\right) & M_{14}^{\prime}\left(r_{2}\right) & M_{15}^{\prime}\left(r_{2}\right) & M_{16}^{\prime}\left(r_{2}\right) \\
M_{21}^{\prime}\left(r_{2}\right) & M_{22}^{\prime}\left(r_{2}\right) & M_{23}^{\prime}\left(r_{2}\right) & M_{24}^{\prime}\left(r_{2}\right) & M_{25}^{\prime}\left(r_{2}\right) & M_{26}^{\prime}\left(r_{2}\right) \\
M_{41}^{\prime}\left(r_{2}\right) & M_{42}^{\prime}\left(r_{2}\right) & M_{43}^{\prime}\left(r_{2}\right) & M_{44}^{\prime}\left(r_{2}\right) & 0 & 0
\end{array}\right|,
$$

The terms $M_{i j}(r)$ appearing in $D_{1}$ and $D_{2}$ are given in Appendix A for $k=0$. It clears that, for $k=0, \alpha_{1}^{2}, \alpha_{2}^{2}, \alpha_{3}^{2}$ being all positive, Bessel functions of the first and second kind enter the solution. Equation (30) is satisfied, if either $D_{1}$ or $D_{2}$ is equal to zero. The case of $D_{1}=0$ corresponds to plane-strain vibrations of thick-walled hollow poroelastic cylinders for a pervious surface.

The case of $D_{2}=0$, corresponds to longitudinal vibrations which involve only longitudinal displacement $w$. Also, $D_{2}=0$ is the same for a pervious and an impervious surface; hence, it is clear that longitudinal shear vibrations are independent of the nature of the surface. From (27), it is clear that plane-strain vibrations and longitudinal shear vibrations are uncoupled for a pervious surface, when the motion is independent of longitudinal coordinated $z$, and these vibrations are coupled for nonzero longitudinal wave number $k$; that is, $k \neq 0$. The frequency equation of longitudinal shear vibrations $D_{2}=0$, when expanded, gives

$$
J_{n}^{\prime}\left(\alpha_{3} r_{1}\right) Y_{n}^{\prime}\left(\alpha_{3} r_{2}\right)-J_{n}^{\prime}\left(\alpha_{3} r_{2}\right) Y_{n}^{\prime}\left(\alpha_{3} r_{1}\right)=0
$$

And the amplitude ratio is given as

$$
\frac{A_{1}}{B_{1}}=-\frac{Y_{n}^{\prime}\left(\alpha_{3} r_{1}\right)}{J_{n}^{\prime}\left(\alpha_{3} r_{1}\right)},
$$

the frequency equation of longitudinal shear vibrations of hollow poroelastic cylinders (31).

4.2. Motion Independent of $\theta$. When the motion is independent of angular coordinated $\theta$ (i.e., $n=0$ ), the frequency equation (27) for a pervious surface is reduced to the product of two determinants given by

$$
D_{3} D_{4}=0 \text {, }
$$

where

$$
D_{3}=\left|\begin{array}{cccccc}
M_{11}^{\prime \prime}\left(r_{1}\right) & M_{12}^{\prime \prime}\left(r_{1}\right) & M_{13}^{\prime \prime}\left(r_{1}\right) & M_{14}^{\prime \prime}\left(r_{1}\right) & M_{17}^{\prime \prime}\left(r_{1}\right) & M_{18}^{\prime \prime}\left(r_{1}\right) \\
M_{31}^{\prime \prime}\left(r_{1}\right) & M_{32}^{\prime \prime}\left(r_{1}\right) & M_{33}^{\prime \prime}\left(r_{1}\right) & M_{34}^{\prime \prime}\left(r_{1}\right) & M_{37}^{\prime \prime}\left(r_{1}\right) & M_{38}^{\prime \prime}\left(r_{1}\right) \\
M_{41}^{\prime \prime}\left(r_{1}\right) & M_{42}^{\prime \prime}\left(r_{1}\right) & M_{43}^{\prime \prime}\left(r_{1}\right) & M_{44}^{\prime \prime}\left(r_{1}\right) & 0 & 0 \\
M_{11}^{\prime \prime}\left(r_{2}\right) & M_{12}^{\prime \prime}\left(r_{2}\right) & M_{13}^{\prime \prime}\left(r_{2}\right) & M_{14}^{\prime \prime}\left(r_{2}\right) & M_{17}^{\prime \prime}\left(r_{2}\right) & M_{18}^{\prime \prime}\left(r_{2}\right) \\
M_{31}^{\prime \prime}\left(r_{2}\right) & M_{32}^{\prime \prime}\left(r_{2}\right) & M_{33}^{\prime \prime}\left(r_{2}\right) & M_{34}^{\prime \prime}\left(r_{2}\right) & M_{37}^{\prime \prime}\left(r_{2}\right) & M_{38}^{\prime \prime}\left(r_{2}\right) \\
M_{41}^{\prime \prime}\left(r_{2}\right) & M_{42}^{\prime \prime}\left(r_{2}\right) & M_{43}^{\prime \prime}\left(r_{2}\right) & M_{44}^{\prime \prime}\left(r_{2}\right) & 0 & 0
\end{array}\right|
$$


The terms $M_{i j}^{\prime}(r)$ in $D_{3}$ and $D_{4}$ are given in Appendix B for $n=0$. From (33), now (32) is satisfied if $D_{3}=0$ or $D_{4}=0$. The case of $D_{3}=0$ gives the frequency equation of axial symmetric vibrations of an infinite hollow poroelastic cylinder for a pervious surface discussed by Ahmed Shah [37].

The case of $D_{4}=0$ when simplified yields the equation

$$
J_{2}\left(\alpha_{3} r_{1}\right) Y_{2}\left(\alpha_{3} r_{2}\right)-J_{2}\left(\alpha_{3} r_{2}\right) Y_{2}\left(\alpha_{3} r_{1}\right)=0,
$$

which is the frequency of torsional vibrations of infinite hollow poroelastic cylinder studied by El-Naggar et al. [2] in the presence of dissipation. Moreover, the frequency equation (34) is independent of the nature of the surface, that is, pervious.

Also, it can be said that (34) is the same for pervious surfaces.

4.3. Equivoluminal Modes. For $n=0$, the stress free boundary conditions on the inner and outer surfaces of the hollow poroelastic cylinder are satisfied if

$$
\alpha_{3}^{2}=k^{2}>0
$$

The dilatational and equivoluminal potentials $f_{1}$ and $g_{1}$ are coupled through the boundary conditions. In particular, to consider purely equivoluminal modes, we set

$$
f_{1}=f_{2}=0 \quad g_{3}=0 .
$$

Using (36) into (17), it is seen that the dilatations of solid and liquid media are zero. Hence, from (2), the liquid pressure is zero. Therefore, the equivoluminal modes are independent of the nature of surface, that is, pervious and impervious. Accordingly, no distinctions between pervious surfaces are seen. Therefore, from (35) and (36), we have

$$
g_{1}^{\prime}\left(\alpha_{3} r_{1}\right)=g_{1}^{\prime}\left(\alpha_{3} r_{2}\right)=0 \quad \sigma_{r r}+s=0,
$$

where

$$
g_{1}\left(\alpha_{3} r\right)=A_{1} J_{1}\left(\alpha_{3} r\right)+B_{1} Y_{1}\left(\alpha_{3} r\right) .
$$

Substituting from (38) into (37) and eliminating the constants $A_{1}, B_{1}$, the frequency equation of purely equivoluminal modes is

$$
J_{1}^{\prime}\left(\alpha_{3} r_{1}\right) Y_{1}^{\prime}\left(\alpha_{3} r_{2}\right)-J_{1}^{\prime}\left(\alpha_{3} r_{2}\right) Y_{1}^{\prime}\left(\alpha_{3} r_{1}\right)=0,
$$

with the amplitude ratio

$$
\frac{A_{1}}{B_{1}}=-\frac{Y_{1}^{\prime}\left(\alpha_{3} r_{1}\right)}{J_{1}^{\prime}\left(\alpha_{3} r_{1}\right)}
$$

And the nonzero displacement and stress for equivoluminal modes are

$$
\begin{aligned}
U & =i k\left[A_{1} J_{1}\left(\alpha_{3} r_{1}\right)+B_{1} Y_{1}\left(\alpha_{3} r_{2}\right)\right] e^{i(k z+w t)} \\
W & =-\alpha_{3}\left[A_{1} J_{0}\left(\alpha_{3} r\right)+B_{1} Y_{0}\left(\alpha_{3} r\right)\right] e^{i(k z+w t)} \\
\sigma_{r r} & =-2 N k i\left[A_{1} J_{1}^{\prime}\left(\alpha_{3} r\right)+B_{1} Y_{1}^{\prime}\left(\alpha_{3} r\right)\right] e^{i(k z+w t)} .
\end{aligned}
$$

Equation (39) is the same as (34) of longitudinal shear vibrations for a case of $n=1$.

4.3.1. For Thin Poroelastic Cylindrical Shell. When $h / r_{1} \ll 1$, that is, for thin poroelastic cylindrical shell, the frequency equation (27) by using Hankel-Kirchhoff asymptotic approximations [38] is reduced to

$$
\operatorname{Sin}\left(\alpha_{3} h\right)-\frac{7 \alpha_{3} h}{8 \alpha_{3} r_{1} r_{2}} \cos \left(\alpha_{3} h\right)+\frac{49}{64 \alpha_{3} r_{1} r_{2}} \sin \left(\alpha_{3} h\right) \approx 0 .
$$

As $\alpha_{3} r_{1} \rightarrow \infty, \alpha_{3} r_{2} \rightarrow \infty$, with the help of (41), (27) is simplified to

$$
\omega \approx \sqrt{2} \frac{q \pi v_{3}}{h}\left[1+\frac{7}{8 q^{2} \pi^{2}}\left(\frac{h}{r_{1}}\right)^{2}\right], \quad(q=1,2,3, \ldots) .
$$

Equation (43) determines the frequency of purely equivoluminal modes of a poroelastic plate of thickness $h$.

4.3.2. For Poroelastic Solid Cylinder. When $r_{1} / h \rightarrow 0$, that is, for the poroelastic solid cylinder, the frequency equation (39) is reduced asymptotically to

$$
J_{1}^{\prime}\left(\alpha_{3} r\right)=0
$$

which is the frequency equation of purely equivoluminal modes of a poroelastic solid cylinder of radius $h$.

\section{Nondimensional Frequency Equation}

For propagating modes in a nondissipative medium, the wave number $k$ is real. To analyze the frequency equation of pervious and impervious surfaces, it is convenient to introduce the following nondimensional parameters:

$$
\begin{aligned}
& P^{\prime}=\frac{P}{H}, \quad Q^{\prime}=\frac{Q}{H}, \\
& R^{\prime}=\frac{R}{H}, \quad N^{\prime}=\frac{N}{H}, \\
& \rho_{11}^{\prime}=\frac{\rho_{11}}{\rho}, \quad \rho_{12}^{\prime}=\frac{\rho_{12}}{\rho}, \\
& \rho_{22}^{\prime}=\frac{\rho_{22}}{\rho}, \quad W=\omega h C_{0}^{-1}, \\
& \varepsilon_{1}=\frac{V_{0}^{2}}{V_{1}^{2}}, \quad \varepsilon_{2}=\frac{V_{0}^{2}}{V_{2}^{2}}, \\
& \delta=\frac{h}{L}, \quad \varepsilon_{3}=\frac{V_{0}^{2}}{V_{3}^{2}},
\end{aligned}
$$

where $w$ is nondimensional frequency,

$$
H=P+2 Q+R, \quad \rho=\rho_{11}+2 \rho_{12}+\rho_{22},
$$

where $C_{0}$ and $V_{0}$ are the reference velocities taking the following form

$$
\left(C_{0}^{2}=\frac{N}{\rho}, V_{0}^{2}=\frac{H}{\rho}\right)
$$



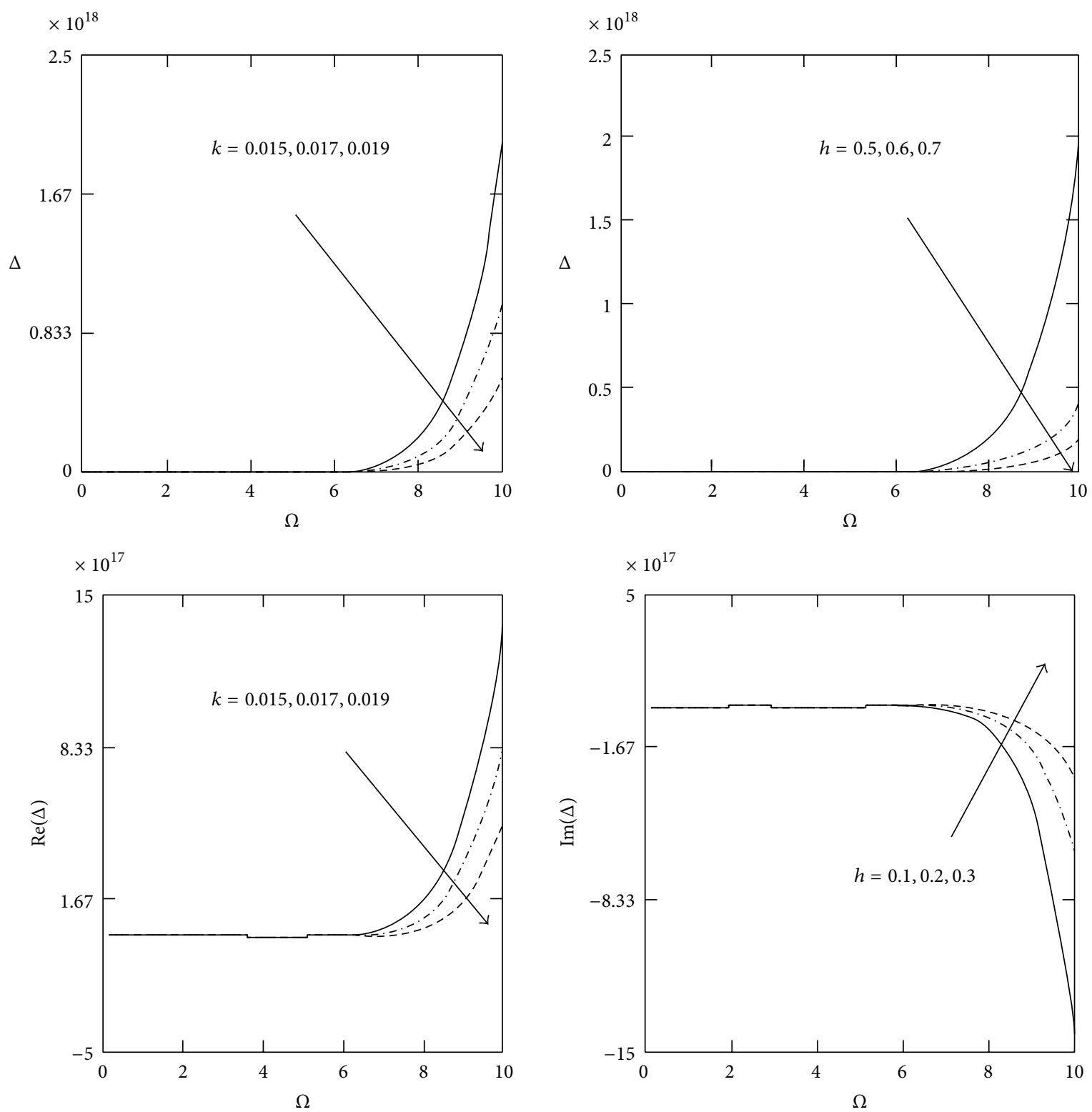

FIGURE 1: Effect of the frequency determinant $\Delta$ with respect to the rotation $\Omega$ for different values of $k, h$, and $n$.

where $h$ is the thickness of the hollow poroelastic cylinder and $L$ is the wavelength.

Let

$$
g=\frac{r_{2}}{r_{1}} \quad \text { so that } \frac{h}{r_{1}}=(g-1) \text {. }
$$

\section{Numerical Results and Discussion}

The numerical results for frequency equations are computed for the poroelastic material. Since the frequency equation, phase velocity, and attenuation coefficient are transcendental in nature. The roots are obtained for $n=0$, the axisymmetric mode, and for the flexural mode $n=1$. The values of the elastic constants of poroelastic material, one being sandstone saturated with kerosene [37] and the other one being sandstone with water [39], are as follows:

$$
\begin{aligned}
& P^{\prime}=0.843, \quad Q^{\prime}=0.065, \quad R^{\prime}=0.028, \quad N^{\prime}=0.234 \\
& \rho_{11}^{\prime}=0.901, \quad \rho_{12}^{\prime}=0.001, \quad \rho_{22}^{\prime}=0.101, \quad \varepsilon_{1}=0.999 \text {, } \\
& \varepsilon_{2}=4.763, \quad \varepsilon_{3}=3.851 \text {. }
\end{aligned}
$$

The numerical technique outlined above was used to obtain frequency equation with respect to rotation $\Omega$ under the effect of wave number and thickness. For the sake of brevity, some computational results are being presented here. The variations are shown in Figures 1-5, respectively.

Figure 1 shows the variation of the frequency equation $\Delta$, phase velocity $\operatorname{Re}(\Delta)$, and attenuation coefficient $\operatorname{Im}(\Delta)$ 

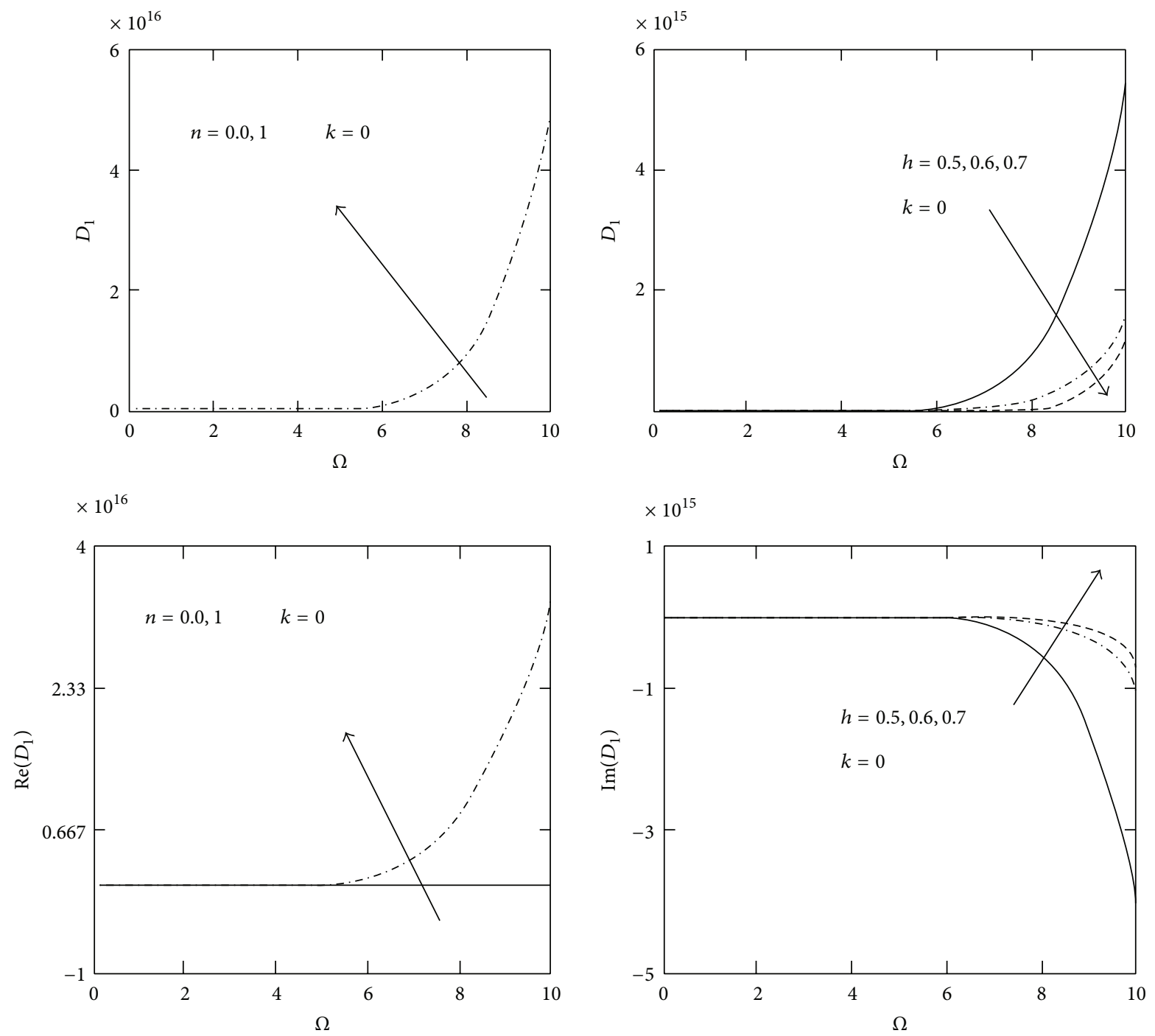

FIGURE 2: Effect of the frequency determinant $D_{1}$ with respect to the rotation $\Omega$ for different values of $n$ and $h$.

of wave propagation in hollow poroelastic circular cylinders with respect to rotation $\Omega$ for different values of wave number $k$ and thickness $h$. In both figures, it is clear that the frequency equation, phase velocity, and attenuation coefficient have a nonzero value only in a bounded region of space. It is observed that the frequency equation and phase velocity increase with increasing rotation, while it decreases with increasing wave number and thickness; as well, attenuation coefficient increases with increasing thickness, while it decreases with increasing of rotation.

Figure 2 shows the variation of the frequency equation $D_{1}$, phase velocity $\operatorname{Re}\left(D_{1}\right)$, and attenuation coefficient $\operatorname{Im}\left(D_{1}\right)$ of wave propagation in hollow poroelastic circular cylinders with respect to rotation $\Omega$ subjected to motion independent of $z$ for different values of longitudinal mode $(n=0)$, flexural mode $(n=1)$, and thickness $h$. In both figures, it is clear that the frequency equation, phase velocity, and attenuation coefficient have a nonzero value only in a bounded region of space. It is observed that the frequency equation and phase velocity increase with increasing rotation, longitudinal mode, and flexural mode, while frequency equation increases with increasing rotation, while it decreases with increasing thickness; as well, attenuation coefficient increases with increasing thickness, while it decreases with increasing rotation.

Figure 3 shows the variation of the frequency equation $D_{2}$ of wave propagation in hollow poroelastic circular cylinders with respect to rotation $\Omega$ subjected to motion independent of $z$ for different values of longitudinal mode $(n=0)$, flexural mode $(n=1)$, and thickness $h$. In both figures, it is clear that the frequency equation has a nonzero value only in a bounded region of space. It is observed that the frequency equation decreases with increasing rotation, while it increases with increasing longitudinal mode and flexural mode; as well, it decreases with increasing of thickness.

Figure 4 shows the variation of the frequency equation $D_{3}$, phase velocity $\operatorname{Re}\left(D_{3}\right)$, and attenuation coefficient $\operatorname{Im}\left(D_{3}\right)$ of wave propagation in hollow poroelastic circular cylinders with respect to rotation $\Omega$ subjected to motion independent 

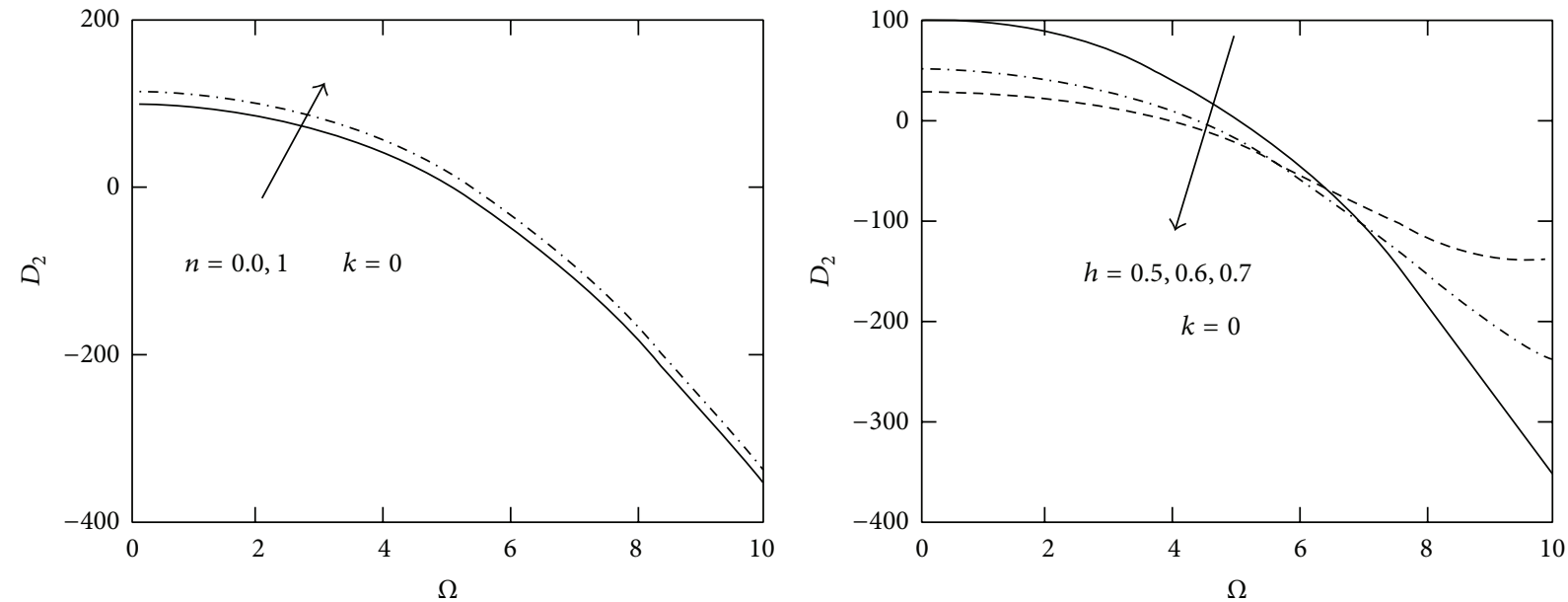

FIgURE 3: Effect of the frequency determinant $D_{2}$ with respect to the rotation $\Omega$ for different values of $h$ and $n$.
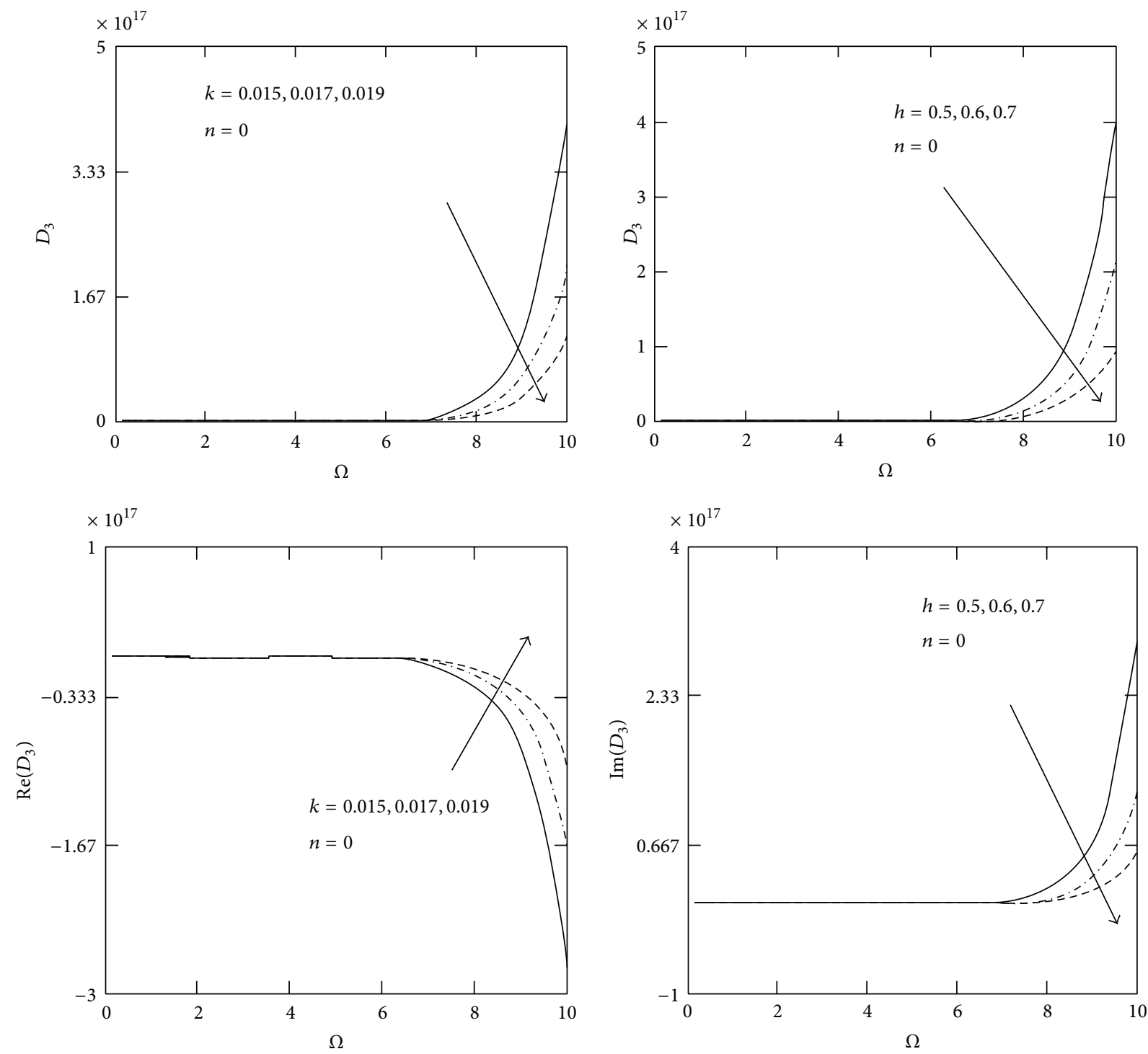

Figure 4: Effect of the frequency determinant $D_{3}$ with respect to the rotation $\Omega$ for different values of $k$ and $h$. 

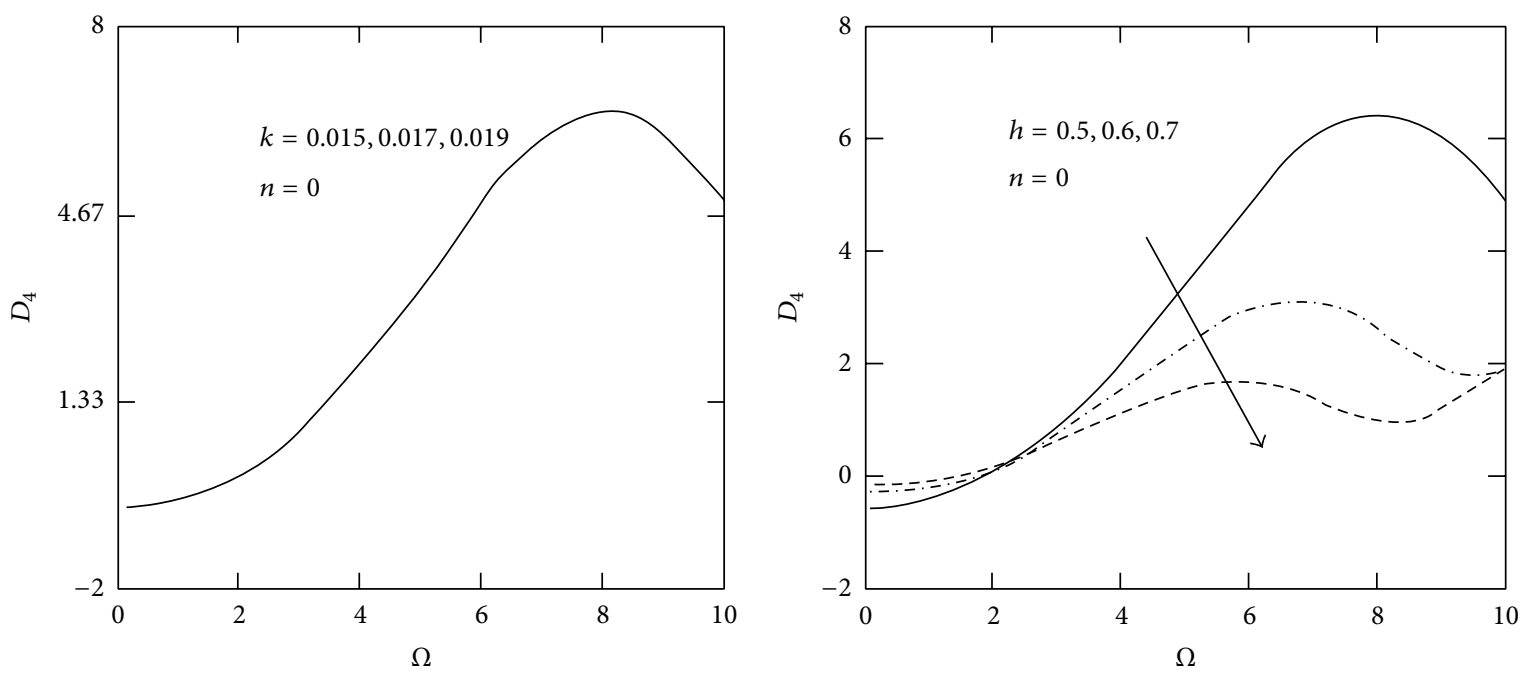

FIGURE 5: Effect of the frequency determinant $D_{4}$ with respect to the rotation $\Omega$ for different values of $k$ and $h$.

of $\theta$ for different values of wave number $k$ and thickness $h$. In both figures, it is clear that the frequency equation, phase velocity, and attenuation coefficient have a nonzero value only in a bounded region of space. It is observed that the frequency equation and attenuation coefficient decrease with increasing wave number and thickness, respectively, while it increases with increasing rotation; as well, attenuation coefficient decreases with increasing thickness, while it increases with increasing rotation.

Figure 5 shows the variation of the frequency equation $D_{4}$ of wave propagation in hollow poroelastic circular cylinders with respect to rotation $\Omega$ subjected to motion independent of $\theta$ for different values of wave number $k$ and thickness $h$. In both figures, it is clear that the frequency equation has a nonzero value only in a bounded region of space. It is observed that the frequency equation decreases with increasing thickness and, while it has oscillatory behavior in the whole range of $\Omega$-axis, there is no effect on the frequency equation.

Comparing with previous studies, we find that our results (shown in Figures 1-5) without rotation for the wave propagation of poroelastic bone with the results obtained by Ahmed Shah [37]. Also, these results agree with those of Kumar [40] by ignoring the liquid and rotation effect and, after rearrangement of terms, the results obtained agree with the purely elastic solid. In case of gravity $\Omega=0$, our results are in agreement with those of Biot [36]. The analytical results obtained by Sharma and Gogna [41] can be considered as a limiting case (by taking $\Omega=0$ ), which are in agreement with earlier results obtained by Fotiadis et al. [42].

\section{Conclusion}

In this paper, the wave propagation of poroelastic bone with circular cylinder subjected to traction free surfaces is considered. We adopted the analysis of [3], and the solution of the problem was expressed in terms of a Bessel function of the first and second kind, respectively.

The resulting frequency equation has been solved numerically. The contribution of the fluid term to wave propagation is a well-established possible mechanism of wave propagation connected to many biological phenomena observed in bone. Although the prediction of the model cannot be trusted quantitatively at this stage, its qualitative behavior complies with the predictions of other theoretical and experimental models referred to in the literature. A calibration of the model and its verification with experimental data is in progress. Important phenomena are observed in all these computations as follows:

(i) the frequency equation of axially symmetric vibrations is independent of the nature of surface, rotation, and presence of fluid in the poroelastic cylindrical;

(ii) by comparing Figures $1-5$, it was found that the frequency equation, phase velocity, and attenuation coefficient have the same behavior in both media; but, with the passage of rotation, wave number, and thickness, numerical values of frequency in the poroelastic cylinder are large in comparison due to the influences of rotation and fluid;

(iii) special cases are considered as motion independent on $z$ and motion independent on $\theta$ in poroelastic medium, as well as in the isotropic case for very large wave numbers and dispersion curves for longitudinal mode and flexural mode $(n=0,1)$, respectively;

(iv) the results presented in this paper should prove to be useful for researchers in material science and designers of new materials and bones;

(v) the study of the phenomenon of rotation, wave number, and thickness is also used to improve the conditions of bones. 


\section{Appendices}

\section{A. Coefficients of $M_{i j}^{\prime}\left(r_{k}\right)$}

The coefficients $M_{i j}^{\prime}\left(r_{k}\right)(i, j=1,2, \ldots, 8, k=1,2)$ are given as follows:

$$
\begin{aligned}
& M_{11}^{\prime}\left(r_{1}\right)=\left\{2 N\left[\frac{n(n-1)}{r_{1}^{2}}-\alpha_{1}^{2}\right]\right. \\
& \left.+\left[(Q+R) \delta_{1}^{2}-(A+Q)\right]\left(\alpha_{1}^{2}\right)\right\} \\
& \times J_{n}\left(\beta_{1} r_{1}\right)+\frac{2 N \beta_{1}}{r_{1}} J_{n+1}\left(\beta_{1} r_{1}\right) \\
& M_{12}^{\prime}\left(r_{1}\right)=\left\{2 N\left[\frac{n(n-1)}{r_{1}^{2}}-\alpha_{1}^{2}\right]\right. \\
& \left.+\left[(Q+R) \delta_{1}^{2}-(A+Q)\right]\left(\alpha_{1}^{2}\right)\right\} \\
& \times Y_{n}\left(\beta_{1} r_{1}\right)+\frac{2 N \beta_{1}}{r} Y_{n+1}\left(\beta_{1} r_{1}\right), \\
& M_{13}^{\prime}\left(r_{1}\right)=\left\{2 N\left[\frac{n(n-1)}{r_{1}^{2}}-\alpha_{1}^{2}\right]\right. \\
& \left.+\left[(Q+R) \delta_{1}^{2}-(A+Q)\right]\left(\alpha_{1}^{2}\right)\right\} \\
& \times J_{n}\left(\beta_{1} r_{1}\right)+\frac{2 N \beta_{1}}{r_{1}} J_{n+1}\left(\beta_{1} r_{1}\right), \\
& M_{14}^{\prime}\left(r_{1}\right)=\left\{2 N\left[\frac{n(n-1)}{r_{1}^{2}}-\alpha_{1}^{2}\right]\right. \\
& \left.+\left[(Q+R) \delta_{1}^{2}-(A+Q)\right]\left(\alpha_{1}^{2}\right)\right\} \\
& \times Y_{n}\left(\beta_{1} r_{1}\right)+\frac{2 N \beta_{1}}{r_{1}} J_{n+1}\left(\beta_{1} r_{1}\right), \\
& M_{15}^{\prime}\left(r_{1}\right)=\frac{2 N n(n-1)}{r_{1}^{2}} J_{n}\left(\beta_{3} r_{1}\right)-\frac{2 N n \beta_{3}}{r_{1}} J_{n+1}\left(\beta_{3} r_{1}\right) \text {, } \\
& M_{16}^{\prime}\left(r_{1}\right)=\frac{2 N n(n-1)}{r_{1}^{2}} Y_{n}\left(\beta_{3} r_{2}\right)-\frac{2 N n \beta_{3}}{r_{1}} Y_{n+1}\left(\beta_{3} r_{1}\right) \text {, } \\
& M_{21}^{\prime}\left(r_{1}\right)=\frac{2 N n(1-n)}{r_{1}^{2}} Y_{n}\left(\beta_{1} r_{1}\right)+\frac{2 N n \beta_{1}}{r} J_{n+1}\left(\beta_{1} r_{1}\right) \text {, } \\
& M_{22}^{\prime}\left(r_{1}\right)=\frac{2 N n(1-n)}{r_{1}^{2}} Y_{n}\left(\beta_{1} r_{1}\right)+\frac{2 N n \beta_{1}}{r} Y_{n+1}\left(\beta_{1} r_{1}\right) \text {, } \\
& M_{23}^{\prime}\left(r_{1}\right)=\frac{2 N n(1-n)}{r_{1}^{2}} J_{n}\left(\beta_{2} r_{1}\right)+\frac{2 N n \beta_{2}}{r_{1}} J_{n+1}\left(\beta_{2} r_{1}\right) \text {, } \\
& M_{24}^{\prime}\left(r_{1}\right)=\frac{2 N n(1-n)}{r_{1}^{2}} Y_{n}\left(\beta_{2} r_{1}\right)+\frac{2 N n \beta_{2}}{r_{1}} Y_{n+1}\left(\beta_{2} r_{1}\right) \text {, }
\end{aligned}
$$$$
M_{25}^{\prime}\left(r_{1}\right)=\left[\frac{2 N n(1-n)}{r_{1}^{2}}+N \alpha_{3}^{2}\right] J_{n}\left(\beta_{3} r_{1}\right)
$$$$
-\frac{2 N \beta_{3}}{r_{1}} J_{n+1}\left(\beta_{3} r_{1}\right) \text {, }
$$$$
M_{26}^{\prime}\left(r_{1}\right)=\left[\frac{2 N n(1-n)}{r_{1}^{2}}+N \alpha_{3}^{2}\right] Y_{n}\left(\beta_{3} r_{1}\right)
$$$$
-\frac{2 N \beta_{3}}{r_{1}} Y_{n+1}\left(\beta_{3} r_{1}\right) \text {, }
$$$$
M_{37}^{\prime}\left(r_{1}\right)=\frac{-N n \beta_{3}}{r_{1}} J_{n}\left(\beta_{3} r_{1}\right)-N\left(\alpha_{3}^{2}\right) J_{n+1}\left(\beta_{3} r_{1}\right) \text {, }
$$$$
M_{38}^{\prime}\left(r_{1}\right)=\frac{-N n \beta_{3}}{r_{1}} Y_{n}\left(\beta_{3} r_{1}\right)-N\left(\alpha_{3}^{2}\right) Y_{n+1}\left(\beta_{3} r_{1}\right),
$$$$
M_{37}^{\prime}\left(r_{2}\right)=\frac{-N n \beta_{3}}{r_{2}} J_{n}\left(\beta_{3} r_{2}\right)-N\left(\alpha_{3}^{2}\right) J_{n+1}\left(\beta_{3} r_{2}\right) \text {, }
$$$$
M_{38}^{\prime}\left(r_{2}\right)=\frac{-N n \beta_{3}}{r_{2}} Y_{n}\left(\beta_{3} r_{2}\right)-N\left(\alpha_{3}^{2}\right) Y_{n+1}\left(\beta_{3} r_{2}\right) \text {, }
$$$$
M_{41}^{\prime}\left(r_{1}\right)=\left(R \delta_{1}^{2}-Q\right)\left(\alpha_{1}^{2}\right) J_{n}\left(\beta_{1} r_{1}\right),
$$$$
M_{42}^{\prime}\left(r_{1}\right)=\left(R \delta_{1}^{2}-Q\right)\left(\alpha_{1}^{2}\right) Y_{n}\left(\beta_{1} r_{1}\right) \text {, }
$$$$
M_{43}^{\prime}\left(r_{1}\right)=\left(R \delta_{2}^{2}-Q\right)\left(\alpha_{1}^{2}\right) J_{n}\left(\beta_{2} r_{1}\right) \text {, }
$$$$
M_{44}^{\prime}\left(r_{1}\right)=\left(R \delta_{2}^{2}-Q\right)\left(\alpha_{2}^{2}\right) J_{n}\left(\beta_{2} r_{1}\right) \text {, }
$$$$
M_{45}^{\prime}\left(r_{1}\right)=0 \text {, }
$$$$
M_{46}^{\prime}\left(r_{1}\right)=0 \text {, }
$$$$
M_{41}^{\prime}\left(r_{2}\right)=\left(R \delta_{1}^{2}-Q\right)\left(\alpha_{1}^{2}\right) J_{n}\left(\beta_{1} r_{2}\right) \text {, }
$$$$
M_{42}^{\prime}\left(r_{2}\right)=\left(R \delta_{1}^{2}-Q\right)\left(\alpha_{1}^{2}\right) Y_{n}\left(\beta_{1} r_{2}\right),
$$$$
M_{43}^{\prime}\left(r_{2}\right)=\left(R \delta_{2}^{2}-Q\right)\left(\alpha_{1}^{2}\right) J_{n}\left(\beta_{2} r_{2}\right) \text {, }
$$$$
M_{44}^{\prime}\left(r_{2}\right)=\left(R \delta_{2}^{2}-Q\right)\left(\alpha_{2}^{2}\right) J_{n}\left(\beta_{2} r_{2}\right) \text {, }
$$$$
M_{45}^{\prime}\left(r_{2}\right)=0 \text {, }
$$$$
M_{46}^{\prime}\left(r_{2}\right)=0 \text {. }
$$

\section{B. Coefficients of $M_{i j}^{\prime \prime}\left(r_{k}\right)$}

The coefficients $M_{i j}^{\prime \prime}\left(r_{k}\right)(i, j=1,2, \ldots, 8, k=1,2)$ are given as follows:

$$
\begin{aligned}
M_{11}^{\prime \prime}\left(r_{1}\right)=\{ & -2 N \alpha_{1}^{2}+\left[(Q+R) \delta_{1}^{2}-(A+Q)\right] \\
& \left.\times\left(k^{2}+\alpha_{1}^{2}\right)\right\} \\
& \times J_{n}\left(\beta_{1} r_{1}\right)+\frac{2 N \beta_{1}}{r_{1}} J_{n+1}\left(\beta_{1} r_{1}\right),
\end{aligned}
$$




$$
\begin{aligned}
& M_{12}^{\prime \prime}\left(r_{1}\right)=\left\{-2 N \alpha_{1}^{2}+\left[(Q+R) \delta_{1}^{2}-(A+Q)\right]\right. \\
& \left.\times\left(k^{2}+\alpha_{1}^{2}\right)\right\} \\
& \times Y_{n}\left(\beta_{1} r_{1}\right)+\frac{2 N \beta_{1}}{r_{1}} Y_{n+1}\left(\beta_{1} r_{1}\right), \\
& M_{13}^{\prime \prime}\left(r_{1}\right)=\left\{-2 N \alpha_{2}^{2}+\left[(Q+R) \delta_{2}^{2}-(A+Q)\right]\right. \\
& \left.\times\left(k^{2}+\alpha_{2}^{2}\right)\right\} \\
& \times J_{n}\left(\beta_{2} r_{1}\right)+\frac{2 N \beta_{2}}{r_{1}} J_{n+1}\left(\beta_{2} r_{1}\right), \\
& M_{14}^{\prime \prime}\left(r_{1}\right)=\left\{-2 N \alpha_{2}^{2}+\left[(Q+R) \delta_{2}^{2}-(A+Q)\right]\right. \\
& \left.\times\left(k^{2}+\alpha_{2}^{2}\right)\right\} \\
& \times Y_{n}\left(\beta_{2} r_{1}\right)+\frac{2 N \beta_{2}}{r_{1}} J_{n+1}\left(\beta_{2} r_{1}\right), \\
& M_{17}^{\prime \prime}\left(r_{1}\right)=2 N i k \beta_{3} J_{n}\left(\beta_{3} r_{1}\right)-\frac{2 N i k}{r_{1}} J_{n+1}\left(\beta_{3} r_{1}\right) \text {, } \\
& M_{18}^{\prime \prime}\left(r_{1}\right)=2 N i k \beta_{3} Y_{n}\left(\beta_{3} r_{1}\right)-\frac{2 N i k}{r_{1}} Y_{n+1}\left(\beta_{3} r_{1}\right) \text {, } \\
& M_{25}^{\prime \prime}\left(r_{1}\right)=-\frac{2 N \beta_{3}}{r_{1}} J_{n+1}\left(\beta_{3} r_{1}\right) \text {, } \\
& M_{26}^{\prime \prime}\left(r_{1}\right)=\left[N \alpha_{3}^{2}\right] Y_{n}\left(\beta_{3} r_{1}\right)-\frac{2 N \beta_{3}}{r_{1}} Y_{n+1}\left(\beta_{3} r_{1}\right) \text {, } \\
& M_{25}^{\prime \prime}\left(r_{2}\right)=-\frac{2 N \beta_{3}}{r_{2}} J_{n+1}\left(\beta_{3} r_{2}\right) \text {, } \\
& M_{26}^{\prime \prime}\left(r_{2}\right)=\left[N \alpha_{3}^{2}\right] Y_{n}\left(\beta_{3} r_{2}\right)-\frac{2 N \beta_{3}}{r_{1}} Y_{n+1}\left(\beta_{3} r_{2}\right) \text {, } \\
& M_{31}^{\prime \prime}\left(r_{1}\right)=-2 N i k \beta_{1} J_{n+1}\left(\beta_{1} r_{1}\right), \\
& M_{32}^{\prime \prime}\left(r_{1}\right)=-2 N i k \beta_{1} Y_{n+1}\left(\beta_{1} r_{1}\right) \text {, } \\
& M_{33}^{\prime \prime}\left(r_{1}\right)=-2 N i k \beta_{2} J_{n+1}\left(\beta_{2} r_{1}\right) \text {, } \\
& M_{34}^{\prime \prime}\left(r_{1}\right)=-2 N i k \beta_{2} Y_{n+1}\left(\beta_{2} r_{1}\right), \\
& M_{37}^{\prime \prime}\left(r_{1}\right)=-N\left(k^{2}-\alpha_{3}^{2}\right) J_{n+1}\left(\beta_{3} r_{1}\right), \\
& M_{38}^{\prime \prime}\left(r_{1}\right)=-N\left(k^{2}-\alpha_{3}^{2}\right) Y_{n+1}\left(\beta_{3} r_{1}\right), \\
& M_{41}^{\prime \prime}\left(r_{1}\right)=\left(R \delta_{1}^{2}-Q\right)\left(k^{2}+\alpha_{1}^{2}\right) J_{n}\left(\beta_{1} r_{1}\right), \\
& M_{42}^{\prime \prime}\left(r_{1}\right)=\left(R \delta_{1}^{2}-Q\right)\left(k^{2}+\alpha_{1}^{2}\right) Y_{n}\left(\beta_{1} r_{1}\right), \\
& M_{43}^{\prime \prime}\left(r_{1}\right)=\left(R \delta_{2}^{2}-Q\right)\left(k^{2}+\alpha_{1}^{2}\right) J_{n}\left(\beta_{2} r_{1}\right), \\
& M_{44}^{\prime \prime}\left(r_{1}\right)=\left(R \delta_{2}^{2}-Q\right)\left(k^{2}+\alpha_{2}^{2}\right) J_{n}\left(\beta_{2} r_{1}\right) \text {, } \\
& M_{47}^{\prime \prime}\left(r_{1}\right)=0 \text {, }
\end{aligned}
$$

$$
\begin{aligned}
& M_{48}^{\prime \prime}\left(r_{1}\right)=0, \\
M_{11}^{\prime \prime}\left(r_{2}\right)=\{ & -2 N \alpha_{1}^{2}+\left[(Q+R) \delta_{1}^{2}-(A+Q)\right] \\
& \left.\times\left(k^{2}+\alpha_{1}^{2}\right)\right\} \\
\times & J_{n}\left(\beta_{1} r_{2}\right)+\frac{2 N \beta_{1}}{r_{2}} J_{n+1}\left(\beta_{1} r_{2}\right), \\
M_{12}^{\prime \prime}\left(r_{2}\right)= & \left\{2 N \alpha_{1}^{2}+\left[(Q+R) \delta_{1}^{2}-(A+Q)\right]\right. \\
& \left.\times\left(k^{2}+\alpha_{1}^{2}\right)\right\} \\
& \times Y_{n}\left(\beta_{1} r_{2}\right)+\frac{2 N \beta_{1}}{r_{2}} Y_{n+1}\left(\beta_{1} r_{2}\right), \\
M_{13}^{\prime \prime}\left(r_{2}\right)=\{- & 2 N \alpha_{2}^{2}+\left[(Q+R) \delta_{2}^{2}-(A+Q)\right] \\
& \left.\times\left(k^{2}+\alpha_{2}^{2}\right)\right\} \\
& \times J_{n}\left(\beta_{2} r_{2}\right)+\frac{2 N \beta_{2}}{r_{2}} J_{n+1}\left(\beta_{2} r_{2}\right), \\
M_{14}^{\prime \prime}\left(r_{2}\right)=\{ & -2 N \alpha_{2}^{2}+\left[(Q+R) \delta_{2}^{2}-(A+Q)\right] \\
& \left.\times\left(k^{2}+\alpha_{2}^{2}\right)\right\} \\
\times & Y_{n}\left(\beta_{2} r_{2}\right)+\frac{2 N \beta_{2}}{r_{2}} J_{n+1}\left(\beta_{2} r_{2}\right),
\end{aligned}
$$$$
M_{17}^{\prime \prime}\left(r_{2}\right)=2 N i k \beta_{3} J_{n}\left(\beta_{3} r_{2}\right)-\frac{2 N i k}{r_{2}} J_{n+1}\left(\beta_{3} r_{2}\right) \text {, }
$$$$
M_{18}^{\prime \prime}\left(r_{2}\right)=2 N i k \beta_{3} Y_{n}\left(\beta_{3} r_{2}\right)-\frac{2 N i k}{r_{2}} Y_{n+1}\left(\beta_{3} r_{2}\right),
$$$$
M_{31}^{\prime \prime}\left(r_{2}\right)=-2 N i k \beta_{1} J_{n+1}\left(\beta_{1} r_{2}\right),
$$$$
M_{32}^{\prime \prime}\left(r_{2}\right)=-2 N i k \beta_{1} Y_{n+1}\left(\beta_{1} r_{2}\right) \text {, }
$$$$
M_{33}^{\prime \prime}\left(r_{2}\right)=-2 N i k \beta_{2} J_{n+1}\left(\beta_{2} r_{2}\right) \text {, }
$$$$
M_{34}^{\prime \prime}\left(r_{2}\right)=-2 N i k \beta_{2} Y_{n+1}\left(\beta_{2} r_{2}\right) \text {, }
$$$$
M_{37}^{\prime \prime}\left(r_{2}\right)=-N\left(k^{2}-\alpha_{3}^{2}\right) J_{n+1}\left(\beta_{3} r_{2}\right),
$$$$
M_{38}^{\prime \prime}\left(r_{2}\right)=-N\left(k^{2}-\alpha_{3}^{2}\right) Y_{n+1}\left(\beta_{3} r_{2}\right),
$$$$
M_{41}^{\prime \prime}\left(r_{2}\right)=\left(R \delta_{1}^{2}-Q\right)\left(k^{2}+\alpha_{1}^{2}\right) J_{n}\left(\beta_{1} r_{2}\right) \text {, }
$$$$
M_{42}^{\prime \prime}\left(r_{2}\right)=\left(R \delta_{1}^{2}-Q\right)\left(k^{2}+\alpha_{1}^{2}\right) Y_{n}\left(\beta_{1} r_{2}\right) \text {, }
$$$$
M_{43}^{\prime \prime}\left(r_{2}\right)=\left(R \delta_{2}^{2}-Q\right)\left(k^{2}+\alpha_{1}^{2}\right) J_{n}\left(\beta_{2} r_{2}\right),
$$$$
M_{44}^{\prime \prime}\left(r_{2}\right)=\left(R \delta_{2}^{2}-Q\right)\left(k^{2}+\alpha_{2}^{2}\right) J_{n}\left(\beta_{2} r_{2}\right),
$$$$
M_{47}^{\prime \prime}\left(r_{2}\right)=0 \text {, }
$$$$
M_{48}^{\prime \prime}\left(r_{2}\right)=0 \text {. }
$$ 


\section{Conflict of Interests}

The authors declare that there is no conflict of interests regarding the publication of this paper.

\section{References}

[1] S. M. Ahmed and A. M. Abd-Alla, "Electromechanical wave propagation in a cylindrical poroelastic bone with cavity," Applied Mathematics and Computation, vol. 133, no. 2-3, pp. 257-286, 2002.

[2] A. M. El-Naggar, A. M. Abd-Alla, and S. R. Mahmoud, "Analytical solution of electro-mechanical wave propagation in long bones," Applied Mathematics and Computation, vol. 119, no. 1, pp. 77-98, 2001.

[3] A. M. Abd-Alla, S. M. Abo-Dahab, and S. R. Mahmoud, "Wave propagation modeling in cylindrical human long wet bones with cavity," Meccanica, vol. 46, no. 6, pp. 1413-1428, 2011.

[4] R. T. Hart, "A theoretical study on the influence of bone maturation rate on surface remodeling predictions: idealized models," Journal of Biomechanics, vol. 23, no. 3, pp. 241-257, 1990.

[5] Q.-H. Qin, C. Qu, and J. Ye, "Thermoelectroelastic solutions for surface bone remodeling under axial and transverse loads," Biomaterials, vol. 26, no. 33, pp. 6798-6810, 2005.

[6] G. Martínez, J. M. García Aznar, M. Doblaré, and M. Cerrolaza, "External bone remodeling through boundary elements and damage mechanics," Mathematics and Computers in Simulation, vol. 73, no. 1-4, pp. 183-199, 2006.

[7] I. G. Jang and I. Y. Kim, "Computational simulation of simultaneous cortical and trabecular bone change in human proximal femur during bone remodeling," Journal of Biomechanics, vol. 43, no. 2, pp. 294-301, 2010.

[8] M. C. Tsili, "Theoretical solutions for internal bone remodeling of diaphyseal shafts using adaptive elasticity theory," Journal of Biomechanics, vol. 33, no. 2, pp. 235-239, 2000.

[9] S. C. Cowin and K. Firoozbakhsh, "Bone remodeling of diaphysial surfaces under constant load: the theoretical predictions," Journal of Biomechanics, vol. 14, no. 7, pp. 471-484, 1981.

[10] J.-F. Ganghoffer, "A contribution to the mechanics and thermodynamics of surface growth. Application to bone external remodeling," International Journal of Engineering Science, vol. 50, no. 1, pp. 166-191, 2012.

[11] N. A. Sims and J. H. Gooi, "Bone remodeling: multiple cellular interactions required for coupling of bone formation and resorption," Seminars in Cell and Developmental Biology, vol. 19, no. 5, pp. 444-451, 2008.

[12] M. Zumsande, D. Stiefs, S. Siegmund, and T. Gross, "General analysis of mathematical models for bone remodeling," Bone, vol. 48, no. 4, pp. 910-917, 2011.

[13] E. Malachanne, D. Dureisseix, and F. Jourdan, "Numerical model of bone remodeling sensitive to loading frequency through a poroelastic behavior and internal fluid movements," Journal of the Mechanical Behavior of Biomedical Materials, vol. 4, no. 6, pp. 849-857, 2011.

[14] A. Vahdati and G. Rouhi, "A model for mechanical adaptation of trabecular bone incorporating cellular accommodation and effects of microdamage and disuse," Mechanics Research Communications, vol. 36, no. 3, pp. 284-293, 2009.

[15] S. J. Hazelwood, R. Bruce Martin, M. M. Rashid, and J. J. Rodrigo, "A mechanistic model for internal bone remodeling exhibits different dynamic responses in disuse and overload," Journal of Biomechanics, vol. 34, no. 3, pp. 299-308, 2001.

[16] C. Qu, Q.-H. Qin, and Y. Kang, "A hypothetical mechanism of bone remodeling and modeling under electromagnetic loads," Biomaterials, vol. 27, no. 21, pp. 4050-4057, 2006.

[17] V. A. Papathanasopoulou, D. I. Fotiadis, G. Foutsitzi, and C. V. Massalas, "A poroelastic bone model for internal remodeling," International Journal of Engineering Science, vol. 40, no. 5, pp. 511-530, 2002.

[18] M. Mengoni and J. P. Ponthot, "Isotropic continuum damage/repair model for alveolar bone remodeling," Journal of Computational and Applied Mathematics, vol. 234, no. 7, pp. 2036-2045, 2010.

[19] C. Boyle and I. Y. Kim, "Three-dimensional micro-level computational study of Wolff's law via trabecular bone remodeling in the human proximal femur using design space topology optimization," Journal of Biomechanics, vol. 44, no. 5, pp. 935942, 2011.

[20] X. Wang, A. M. Erickson, M. R. Allen, D. B. Burr, R. B. Martin, and S. J. Hazelwood, "Theoretical analysis of alendronate and risedronate effects on canine vertebral remodeling and microdamage," Journal of Biomechanics, vol. 42, no. 7, pp. $938-$ 944, 2009.

[21] M. C. Peterson and M. M. Riggs, "A physiologically based mathematical model of integrated calcium homeostasis and bone remodeling," Bone, vol. 46, no. 1, pp. 49-63, 2010.

[22] Q.-H. Qin and J.-Q. Ye, "Thermoelectroelastic solutions for internal bone remodeling under axial and transverse loads," International Journal of Solids and Structures, vol. 41, no. 9-10, pp. 2447-2460, 2004.

[23] C. Boyle and I. Y. Kim, "Comparison of different hip prosthesis shapes considering micro-level bone remodeling and stressshielding criteria using three-dimensional design space topology optimization," Journal of Biomechanics, vol. 44, no. 9, pp. 1722-1728, 2011.

[24] S. C. Cowin and W. C. Van Buskirk, "Surface bone remodelling induced by a medullary pin," Journal of Biomechanics, vol. 12, no. 4, pp. 269-276, 1979.

[25] M. A. Biot, "General theory of three-dimensional consolidation," Journal of Applied Physics, vol. 12, no. 2, pp. 155-164, 1941.

[26] D. H. Hegedus and S. C. Cowin, "Bone remodeling II: small strain adaptive elasticity," Journal of Elasticity, vol. 6, no. 4, pp. 337-352, 1976.

[27] L. W. Zhang, Z. X. Lei, K. M. Liew, and J. L. Yu, "Static and dynamic of carbon nanotube reinforced functionally graded cylindrical panels," Composite Structures, vol. 111, pp. 205-212, 2014.

[28] L. W. Zhang, P. Zhu, and K. M. Lie, "Thermal buckling of functionally graded plates using a local Kriging meshless method," Composite Structures, vol. 108, pp. 472-492, 2014.

[29] P. Zhu, L. W. Zhang, and K. M. Liew, "Geometrically nonlinear thermomechanical analysis of moderately thick functionally graded plates using a local Petrov-Galerkin approach with moving Kriging interpolation," Composite Structures, vol. 107, pp. 298-314, 2014.

[30] K. M. Liew, Z. X. Lei, J. L. Yu, and L. W. Zhang, "Postbuckling of carbon nanotube-reinforced functionally graded cylindrical panels under axial compression using a meshless approach," Computer Methods in Applied Mechanics and Engineering, vol. 268 , pp. 1-17, 2014 
[31] L. W. Zhang, Z. X. Lei, K. M. Liew, and J. L. Yu, "Large deflection geometrically nonlinear analysis of carbon nanotubereinforced functionally graded cylindrical panels," Computer Methods in Applied Mechanics and Engineerin, vol. 273, pp. 118, 2014.

[32] L. W. Zhang, Y. J. Deng, and K. M. Liew, "An improved elementfree Galerkin method for numerical modeling of the biological population problems," Engineering Analysis with Boundary Elements, vol. 40, pp. 181-188, 2014.

[33] R. J. Cheng, L. W. Zhang, and K. M. Liew, "Modeling of biological population problems using the element-free kp-Ritz method," Applied Mathematics and Computation, vol. 227, pp. 274-290, 2014.

[34] A. M. Abd-Alla and S. M. Abo-Dahab, "Effect of magnetic field on poroelastic bone model for internal remodeling," Applied Mathematics and Mechanics, vol. 34, no. 7, pp. 889-906, 2013.

[35] M. A. Biot, "Theory of elasticity and consolidation for a porous anisotropic solid," Journal of Applied Physics, vol. 26, no. 2, pp. 182-185, 1955.

[36] M. A. Biot, "Theory of propagation of elastic waves in a fluid-saturated porous solid. I: low-frequency range," Acoustical Society of America, vol. 28, pp. 168-178, 1956.

[37] S. Ahmed Shah, "Axially symmetric vibrations of fluid-filled poroelastic circular cylindrical shells," Journal of Sound and Vibration, vol. 318, no. 1-2, pp. 389-405, 2008.

[38] A. Abramowitz and I. A. Stegun, Handbook of Mathematical Functions, National Bureau of Standards, Washington, DC, USA, 1956.

[39] C. H. Yew and P. N. Jogi, "Study of wave motions in fluidsaturated porous rocks," Journal of the Acoustical Society of America, vol. 60, no. 1, pp. 2-8, 1976.

[40] R. Kumar, "Dispersion of axially symmetric waves in empty and fluid-filled cylindrical shells," Acustica, vol. 27, no. 6, pp. 317328, 1972.

[41] M. D. Sharma and M. L. Gogna, "Propagation of elastic waves in a cylindrical bore in a liquid-saturated porous solid," Geophysical Journal International, vol. 103, no. 1, pp. 47-54, 1990.

[42] D. I. Fotiadis, G. Foutsitzi, and C. V. Massalas, "Wave propagation modeling in human long bones," Acta Mechanica, vol. 137, no. 1, pp. 65-81, 1999. 


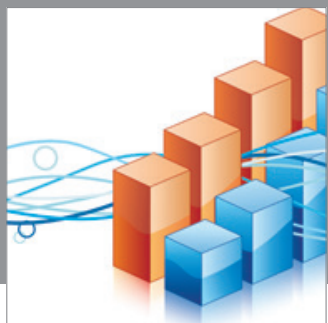

Advances in

Operations Research

mansans

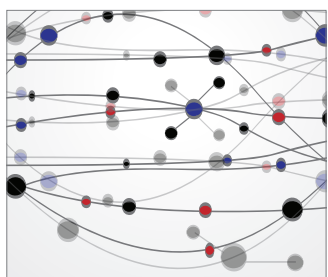

The Scientific World Journal
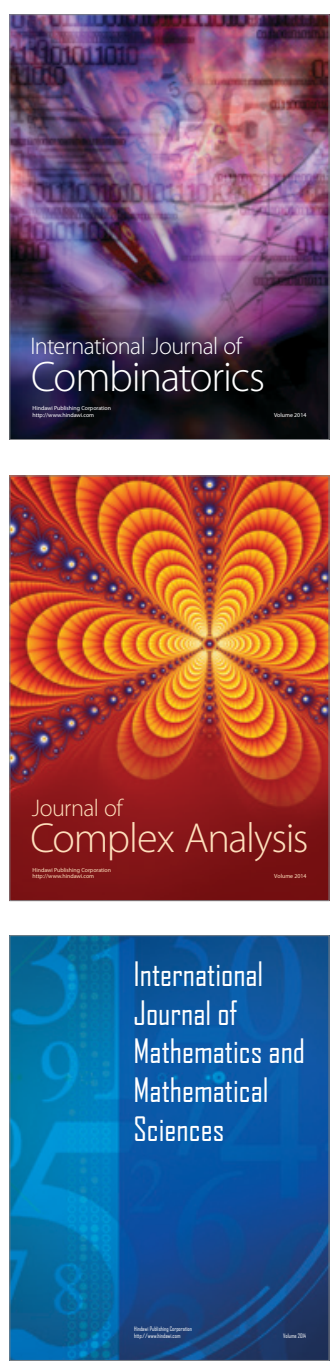
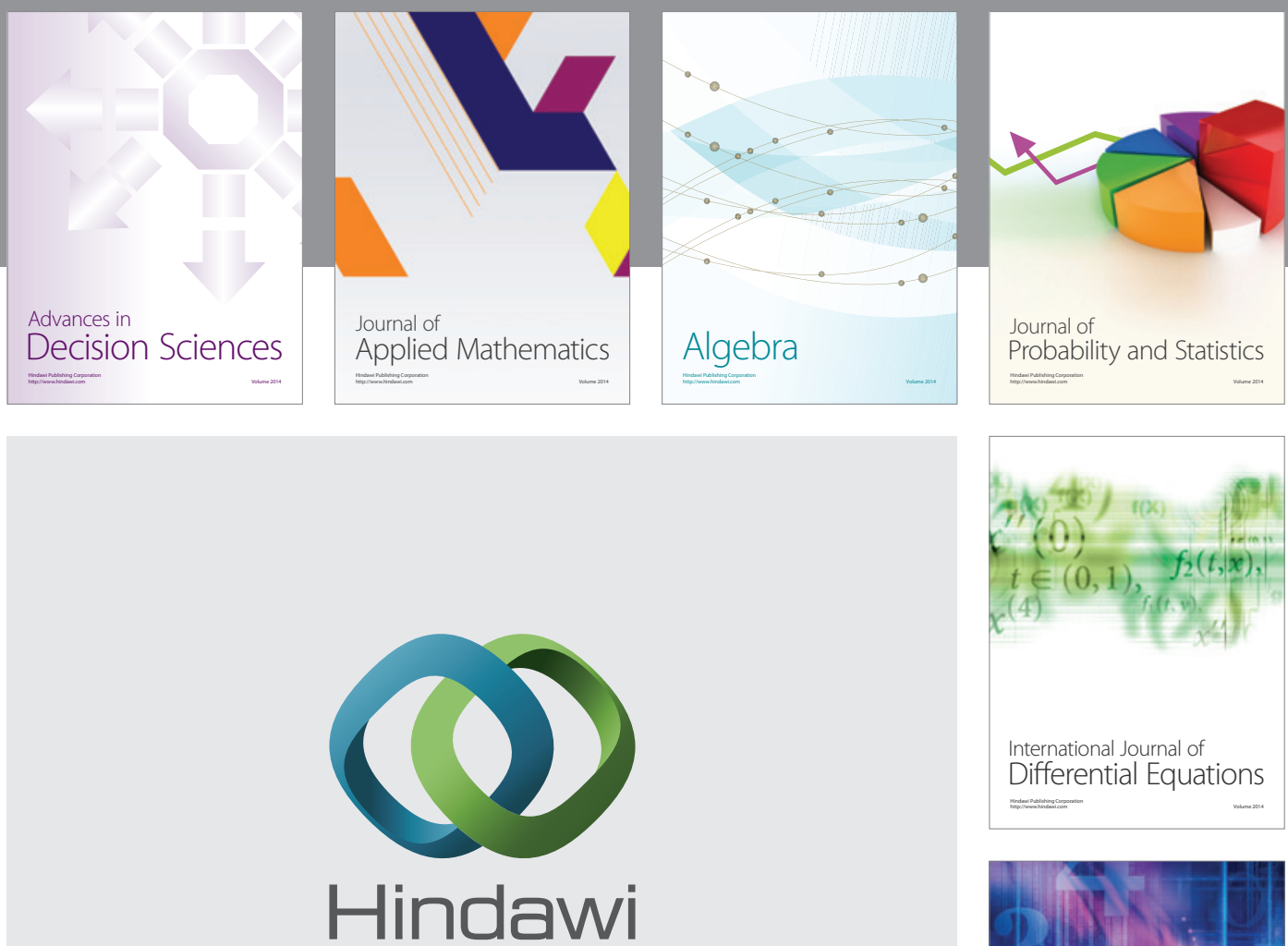

Submit your manuscripts at http://www.hindawi.com
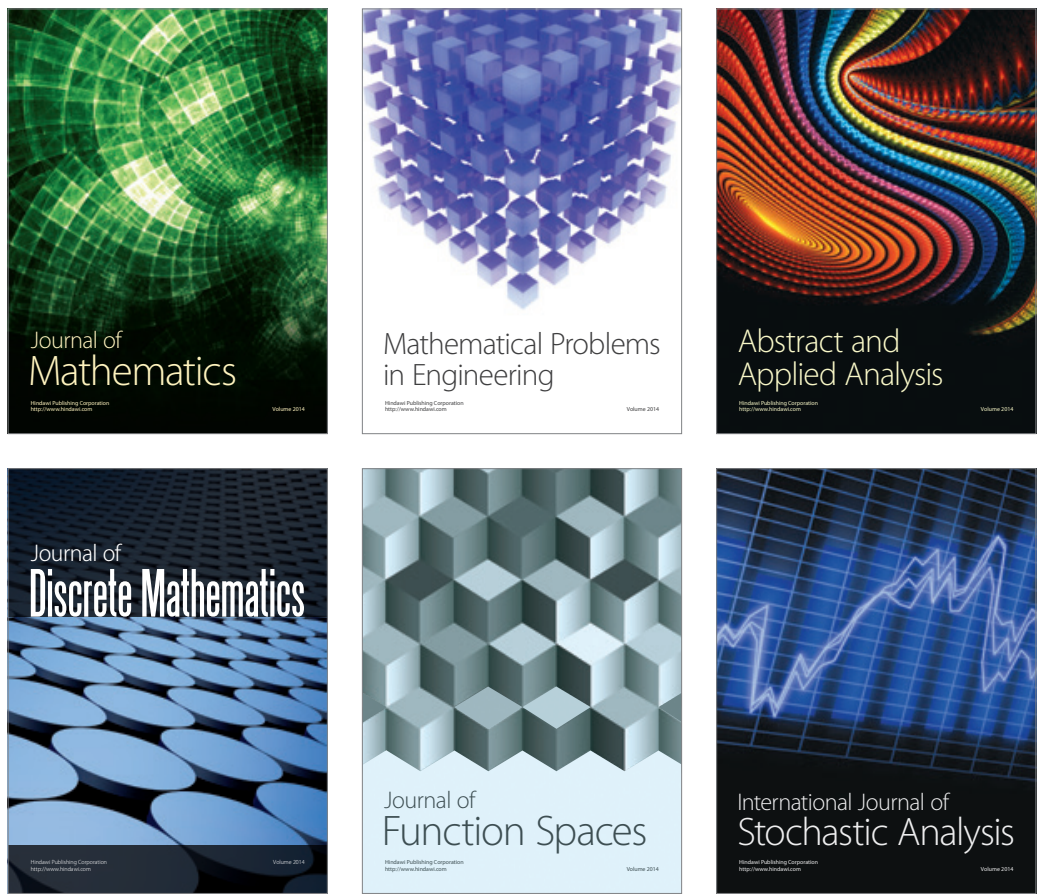

Journal of

Function Spaces

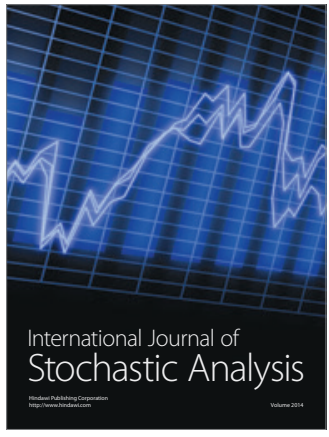

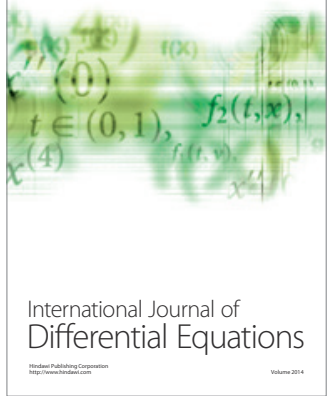
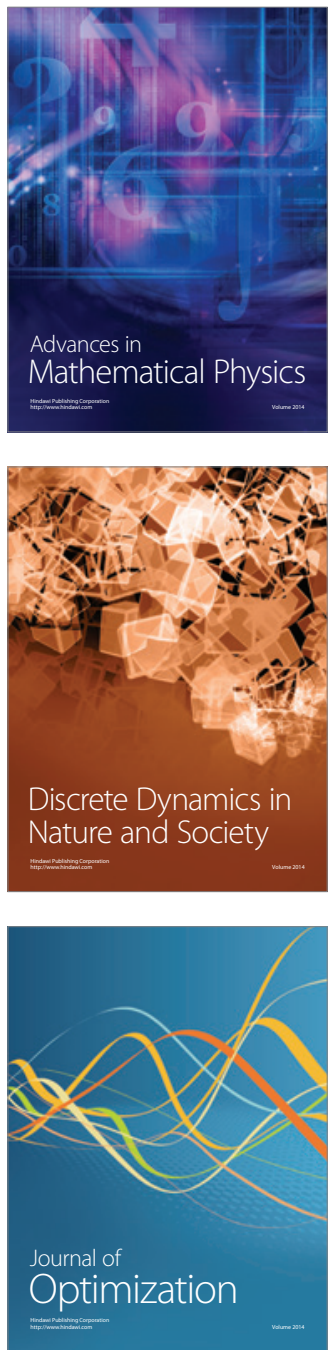